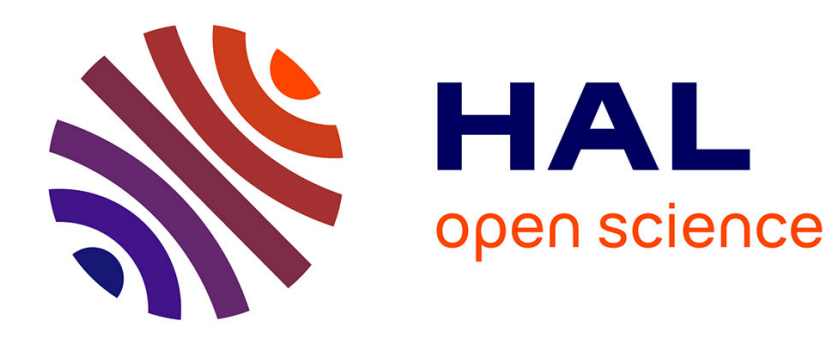

\title{
Causal conformal vector fields, and singularities of twistor spinors \\ Charles Frances
}

\section{To cite this version:}

Charles Frances. Causal conformal vector fields, and singularities of twistor spinors. Annals of Global Analysis and Geometry, 2007, 32 (3), pp.277-295. 10.1007/s10455-007-9060-1 . hal-03165738

\section{HAL Id: hal-03165738 \\ https://hal.science/hal-03165738}

Submitted on 10 Mar 2021

HAL is a multi-disciplinary open access archive for the deposit and dissemination of scientific research documents, whether they are published or not. The documents may come from teaching and research institutions in France or abroad, or from public or private research centers.
L'archive ouverte pluridisciplinaire HAL, est destinée au dépôt et à la diffusion de documents scientifiques de niveau recherche, publiés ou non, émanant des établissements d'enseignement et de recherche français ou étrangers, des laboratoires publics ou privés. 


\title{
Causal conformal vector fields, and singularities of twistor spinors.
}

\author{
Charles Frances
}

\begin{abstract}
In this paper, we study the geometry around the singularity of a twistor spinor, on a Lorentz manifold $(M, g)$ of dimension greater or equal to three, endowed with a spin structure. Using the dynamical properties of conformal vector fields, we prove that the geometry has to be conformally flat on some open subset of any neighbourhood of the singularity. As a consequence, any analytic Lorentz manifold, admitting a twistor spinor with at least one zero has to be conformally flat.
\end{abstract}

Mathematics Subject Classification (2000). 53B30, 53C27, 37C10.

Keywords. Lorentzian conformal geometry, Twistor spinors, Dynamical systems.

\section{Introduction}

Conformal vector fields of a pseudo-Riemannian manifold $(M, g)$ are those vector fields $X$ for which the associated local flow $\phi_{X}^{t}$ acts by conformal transformations. This means that on each open subset $U$ on which $\phi_{X}^{t}$ is defined, $\left(\phi_{X}^{t}\right)^{*} g=e^{2 \sigma_{t}} g$ for some smooth function $\sigma_{t}$ on $U$. When the signature is Lorentzian, i.e $(-,+, \ldots,+)$, the conformal class $[g]$ of $g$ encodes the causal properties of $(M, g)$. In this case, a conformal vector field can be thought as an infinitesimal symmetry of the causal structure on $(M, g)$. That's why such fields were at least as much studied by physicists as by mathematicians. One of the interesting, and natural issues about conformal vector fields, is to describe local normal forms, especially in the neighbourhood of a singularity.

The simplest kind of conformal vector fields are Killing vector fields: those for which $\left(\phi_{X}^{t}\right)^{*} g=g$. In the same category are inessential vector fields, which are Killing fields for a metric $e^{2 \sigma} g$ in the conformal class. Since the flow of these vector fields preserves not just a conformal structure (which are second-order rigid structures), but directly a metric structure (which are rigid of first order), they are fairly well understood. In particular, such vector fields are linearizable in the neighbourhood of any of their singularities. 
In light of this remark, the most interesting conformal vector fields (or at least those which are likely to be quite different from the previous ones) are essential vector fields: the local flow of such vector fields preserves the conformal class $[g]$, but no metric in this class. When a conformal vector field $X$ is given on a manifold, it is not an easy matter to know if it is essential or not. Since on a sufficiently small open set on which $X$ does not vanish, $X$ is always inessential (just push the metric, restricted to a local section, by the local flow $\phi_{X}^{t}$ ), we will now suppose that $X$ has at least one singularity on $X$. Under this asumption, there are sufficient conditions, which ensure that $X$ is essential. For example, if $X$ is a gradient vector field ([KR1], [KR2]), or if $X$ is a similarity vector field ([A]). In these two cases, the geometry of $[g]$ around a singularity is well understood. For gradient vector fields, the situation is very rigid: the geometry around a singularity has to be conformally flat. On the contrary, it is shown in [A] that some similarity vector fields can preserve "quite a lot" of conformal structures near a singularity, especially non conformally flat ones.

In this article, we focus on Lorentz manifolds $(M, g)$ of dimension $\geq 3$, and conformal vector fields $X$ on $(M, g)$ which are causal. This means that $g(X, X) \leq 0$ everywhere (let us recall that for us, Lorentzian signature means signature $(-,+, \ldots,+))$. As we shall see in section 2.3 , non trivial causal conformal vector fields admitting a singularity are automatically essential. The main result of the article is to prove that for these vector fields, any neighbourhood of a singularity admits an open subset on which the geometry is conformally flat. The precise statement is:

Theorem 1. Let $X$ be a causal conformal vector field on a smooth Lorentz manifold $(M, g)$ of dimension greater or equal to three. Suppose that $X$ is nontrivial, and vanishes at $x_{0} \in M$. Then the geometry is conformally flat on a nonempty open subset of $M$. More precisely, there is a neighbourhood $U$ of $x_{0}$, satisfying exactly one of the two following properties:

(i) The only singularity of $X$ in $U$ is $x_{0}$. In this case, the geometry is conformally flat on the open set $I_{U}^{+}\left[x_{0}\right] \cup I_{U}^{-}\left[x_{0}\right]$.

(ii) The singularities of $X$ in $U$ form a lightlike geodesic $\Delta \subset U$. In this case, the geometry is conformally flat on the open set $\bigcup_{x \in \Delta}\left(I_{U}^{+}[x] \cup I_{U}^{-}[x]\right)$.

In the statement, $I_{U}^{+}\left[x_{0}\right]$ (resp. $I_{U}^{-}\left[x_{0}\right]$ ) denotes the future of $x_{0}$ (resp. the past of $x_{0}$ ) in $U$ (these notions will be defined precisely in section 2).

As an immediate corollary, we get:

Corollary 1. Let $(M, g)$ be an analytic Lorentz manifold, and $X$ a nontrivial causal conformal vector field on $M$. Then if $X$ has a zero, $(M, g)$ is conformally flat.

In the last section of the paper (section 6), we will show that the conclusions of Theorem 1 are in fact sharp. Indeed, one can produce examples 
of Lorentz manifolds $(M, g)$ with a causal conformal vector field $X$ having a singularity $x_{0} \in M$, satisfying condition (i) (resp. condition (ii)) of Theorem 1 , and such that $I_{U}^{+}\left[x_{0}\right] \cup I_{U}^{-}\left[x_{0}\right]$ (resp. $\bigcup_{x \in \Delta}\left(I_{U}^{+}[x] \cup I_{U}^{-}[x]\right)$ ) is the biggest open subset of $U$ on which the geometry is conformally flat. Thus, we can only expect "semi-rigidity" of the geometry near a singularity, and the situation is really intermediate between the case studied in [KR1], [KR2], and that of $[\mathrm{A}]$.

For the readers finding assumptions of Theorem 1 a little bit artificial, we describe now a geometric situation where causal conformal vector fields appear naturally (we refer to $[\mathrm{BL}]$, and references therein for details). Let us consider a Lorentz manifold $(M, g)$, admitting a spin structure (see [LM] for an introduction to spin structures). Let us denote by $\mathcal{S}$ the spin bundle. Two important operators can be defined on the sections of $\mathcal{S}$ : the Dirac operator $D$, and the Twistor operator $P$. Sections $\phi \in \Gamma(\mathcal{S})$ such that $P(\phi)=0$ are called twistor spinor. To each spinor field $\phi \in \Gamma(\mathcal{S})$, it is possible to associate a causal vector field $X_{\phi}$ : the Dirac current associated to $\phi$ (this is very clearly explained in the introduction of $[\mathrm{BL}])$. Moreover, the zeros of the Dirac current $X_{\phi}$ are exactly the zeros of $\phi$. Now, a remarkable property of twistor spinors is that their Dirac current is a conformal vector field for $g$. It thus becomes clear, that Theorem 1 gives some informations about the geometry in the neighbourhood of the singularity of a twistor spinor on a Lorentz manifold. In the analytical framework, we get the following conclusion:

Corollary 2. Let $(M, g)$ be an analytic Lorentz manifold, endowed with a spin structure. If $(M, g)$ admits a nonzero twistor spinor which vanishes somewhere, then $(M, g)$ is conformally flat.

\section{Geometrical aspects}

\subsection{Lightlike conformal geodesics}

Let $(M, g)$ be a Lorentz manifold. We denote by $\nabla$ the Levi-Civita connection associated to the metric $g$. For any curve $\gamma: I \rightarrow M$, we will denote by $[\gamma]$ the geometric support of $\gamma$. A curve $\gamma: I \rightarrow M$, with parameter $s \in I$ is a lightlike geodesic of $(M, g)$ if it satisfies the differential equation $\nabla_{\dot{\gamma}} \dot{\gamma}=0$, and $g(\dot{\gamma}(s), \dot{\gamma}(s))=0, \forall s \in I$. It is well known that if $g_{1}=e^{2 \sigma} g$ is a Lorentzian metric in the conformal class of $g$, then the lightlike geodesics of $g_{1}$ will have the same supports as those of $g$. However, the affine parameters of geodesics of $g_{1}$ generally differ from affine parameters of geodesics of $g$. Now, let $s \mapsto \gamma(s)$ be a lightlike geodesic of $(M, g)$, with affine parameter $s$. A parameter $p=p(s)$ will be said projective if it satisfies the equation:

$$
\{p, s\}=-\frac{2}{n-2} \operatorname{Ric}(\dot{\gamma}(s), \dot{\gamma}(s))
$$


In this equation, Ric denotes the Ricci tensor of the metric $g$ and $\{p, s\}$ is the Schwarzian derivative of $p,\{p, s\}=\frac{p^{\prime \prime \prime}}{p^{\prime}}-\frac{3}{2}\left(\frac{p^{\prime \prime}}{p^{\prime}}\right)^{2}$.

Recall that $\{p, u\}=0$ if and only if $p=h(u)$, where $h$ is an homographic transformation. From the chain rule $\{p, u\}=(\{p, s\}-\{u, s\})\left(\frac{d s}{d u}\right)^{2}$, we infer that if $p$ is a projective parameter and $h$ is an homography, then $h(p)$ is again a projective parameter.

Now, let $g_{1}=e^{2 \sigma} g$ be in the conformal class of $g$. Suppose that we parametrize a lightlike geodesic segment $[\gamma]$ by an affine parameter $s$ with respect to the metric $g$, and by an affine parameter $s_{1}$ with respect to the metric $g_{1}$. If $p=p(s)$ is a projective parameter for $[\gamma]$, associated to $s$, and $p_{1}=p_{1}\left(s_{1}\right)$ is a projective parameter associated to $s_{1}$, one can compute (see e.g $[\mathrm{M}])$ that $\left\{p, p_{1}\right\}=0$. In other words, $p_{1}$ is also a projective parameter associated to $s$ and $p$ is a projective parameter associated to $s_{1}$, so that the class of projective parameters depends only on the conformal class of $g$. From this, we can deduce the:

Lemma 1. Let $(M, g)$ be a Lorentz manifold. Suppose that $\left[\gamma_{1}\right]$ and $\left[\gamma_{2}\right]$ are two lightlike geodesic segments parametrized projectively by $t_{1} \mapsto \gamma_{1}\left(t_{1}\right)$ and $t_{2} \mapsto \gamma_{2}\left(t_{2}\right)$. Let $\phi$ be a conformal transformation of $(M, g)$, such that $\phi\left(\left[\gamma_{1}\right]\right)=\left[\gamma_{2}\right]$. Then there is an homographic transformation $h_{\phi}$ such that $\phi \circ \gamma_{1}\left(s_{1}\right)=\gamma_{2} \circ h_{\phi}\left(s_{2}\right)$.

Proposition 1. Let $(M, g)$ be a Lorentz manifold. Every point $x_{0} \in M$ admits a neighbourhood $U$ such that for any pair of points $x, y \in U$, there is a geodesic segment $[\gamma] \subset U$ joining $x$ and $y$. Moreover, if $[\gamma]$ is lightlike, it can be parametrized projectively.

Proof. We start with a neighbourhood $V$ of $x_{0}$ with compact closure, and fix any Riemannian metric \|\| on $\bar{V}$. We denote by $T_{V}^{1} M$ the subset of $T M$ consisting of those $(x, v)$ such that $x \in V, v \in T_{x} M,\|v\|_{x}=1$ and we say that $(x, v) \in T_{V}^{1} \mathcal{L}$ if moreover $v$ is lightlike. Finding a projective parametrization for a geodesic $\gamma(s)=\exp _{x}(s v)$, where $(x, v) \in T_{V}^{1} \mathcal{L}$ is just solving the differential equation $\{p, s\}=-\frac{2}{n-2} \operatorname{Ric}\left(\exp _{x}(s v), \exp _{x}(s v)\right)$. This equation is of the form $p^{\prime \prime \prime}=F\left(x, v, p, p^{\prime}, p^{\prime \prime}\right)$, for a smooth function $F$. So, for any $(x, v) \in T_{V}^{1} \mathcal{L}$, there is a neighbourhood $U_{(x, v)}$ of $(x, v)$ in $T_{V}^{1} \mathcal{L}$ such that for $\left(x^{\prime}, v^{\prime}\right) \in U_{(x, v)}$, the previous equation has a solution defined on ] $-\epsilon(x, v), \epsilon(x, v)\left[\right.$. Taking a finite covering of $T_{V}^{1} \mathcal{L}$ by neighbourhoods of the form $U_{(x, v)}$, one gets the existence of $\epsilon>0$ such that for all $(x, v) \in T_{V}^{1} \mathcal{L}$, the equation $p^{\prime \prime \prime}=F\left(x, v, p, p^{\prime}, p^{\prime \prime}\right)$ has a solution defined on $]-\epsilon, \epsilon[$. Now it is a standard fact of pseudo-Riemannian geometry that $x_{0}$ admits a neighbourhood $U \subset V$ such that for any pair of points $x, y \in U$, one has $y=\exp _{x}(t v)$, for $v \in T_{U}^{1} M$ and $-\frac{\epsilon}{2}<t<+\frac{\epsilon}{2}$ (with $\epsilon$ as above). Then, the neighbourhood $U$ has the desired properties. 


\subsection{Causality on Lorentz manifolds}

Let us recall very briefly some basic causality notions on a Lorentz manifold. We won't make any proof in this section, and the reader who is not familiar with causality is refered to the very complete [BE]. In the whole article, we will work with causally oriented Lorentz manifolds $(M, g)$. This means that there exists on $M$ a non vanishing vector field $Y$ such that $g(Y, Y)<0$ on $M$. This data yields a well defined notion of future oriented causal vectors: if $u \in T_{x} M$, and $g_{x}(u, u) \leq 0, u$ is said to be future oriented if $g_{x}(Y(x), u) \leq 0$. Now, given a piecewise $C^{1}$ curve $\gamma: I \rightarrow M$, we say that the curve $\gamma$ is causal (resp. chronological) if for any $t \in I$ such that $\gamma^{\prime}(t)$ exists, $g\left(\gamma^{\prime}(t), \gamma^{\prime}(t)\right) \leq 0$ (resp. $g\left(\gamma^{\prime}(t), \gamma^{\prime}(t)\right)<0$ ). The curve is said to be future orientied if $\gamma^{\prime}(t)$ is future oriented for any $t \in I$. Notice that if $\gamma$ is a geodesic of the metric $g$, the sign of $g\left(\gamma^{\prime}(t), \gamma^{\prime}(t)\right)$ remains constant on $I$. So a geodesic is said timelike (resp. lightlike, resp. spacelike) if $g\left(\gamma^{\prime}(0), \gamma^{\prime}(0)\right)<0\left(\right.$ resp. $g\left(\gamma^{\prime}(0), \gamma^{\prime}(0)\right)=0$, resp. $\left.g\left(\gamma^{\prime}(0), \gamma^{\prime}(0)\right)>0\right)$.

Given two points $p$ and $q$ on $M$, we write $p \leqslant q$ (resp. $p \ll q$ ) if there is a causal (resp. chronological) future oriented curve $\gamma$ from $p$ to $q$. We then define $I^{+}[p]=\{x \in M, p \ll x\}$, and $I^{-}[p]=\{x \in M, x \ll p\}$. Similary, we can define $J^{+}[p]=\{x \in M, p \leqslant x\}$, and $J^{-}[p]=\{x \in M, x \leqslant p\}$. Notice that in general $p \notin I^{+}[p]$, and $p \notin I^{-}[p]$. The sets $I^{+}[p]$ and $I^{-}[p]$ are always open in $M$. Finally, we denote by $C[p]$ the lightcone with vertex $p$, namely the set of point $q \in M$ lying on a lightlike geodesic segment passing through $p$. We also introduce the sets $C^{+}[p]=C[p] \cap J^{+}[p]$ (resp. $\left.C^{-}[p]=C[p] \cap J^{-}[p]\right)$. An open set $U \subset M$ is said to be convex if for any pair of points $(p, q)$ in $M$, there is a geodesic segment (for the metric $g$ ) joining $p$ and $q$. It is a standard result that any point of $M$ admits a convex neighbourhood. Given $U$ a convex open set, there is a good notion of induced causal structure on $U$. If $p$ and $q$ are two points in $U$, we will write $p \leqslant_{U} q$ (resp. $p \ll_{U} q$ ) if there is a timelike or lightlike (resp. timelike) future oriented geodesic $\gamma$ from $p$ to $q$, contained in $U$. It makes now sense to define $I_{U}^{+}[p]=\left\{x \in U, p \ll_{U} x\right\}$, and $I_{U}^{-}[p]=\left\{x \in U, x \ll_{U} p\right\}$. One defines in the same way $J_{U}^{+}[p]$ and $J_{U}^{-}[p]$. By $C_{U}^{+}[p]$ (resp. $C_{U}^{-}[p]$ ), we mean the future (resp. past) lightcone of vertex $p$, namely $C_{U}^{+}[p]=\{x \in U, \exists \gamma$ : $[0,1] \rightarrow U, \gamma$ future oriented lightlike geodesic, $\gamma(0)=p, \gamma(1)=x\}$ (resp. $C_{U}^{-}[p]=\{x \in U, \exists \gamma:[0,1] \rightarrow U, \gamma$ past oriented lightlike geodesic, $\gamma(0)=$ $p, \gamma(1)=x\})$.

Shrinking $U$ if necessary, we can assume that $J_{U}^{+}[p]$ and $J_{U}^{-}[p]$ are closed convex sets of $U$, for any $p \in U$. The sets $C_{U}^{+}[p]$ and $C_{U}^{-}[p]$ are also closed. Let us fix $p \in U$. There is a closed subset $K \subset U$ containing $J_{U}^{-}[p]$ in its interior such that $J_{U}^{+}[p] \cap K$ is compact. Now, for $q_{1} \in I_{U}^{-}[p]$ sufficiently close to $p, J_{U}^{+}\left[q_{1}\right]$ is close to $J_{U}^{+}[p]$ and $J_{U}^{+}\left[q_{1}\right] \cap K$ is compact. Now, for $q_{2} \in I_{U}^{+}[p]$ sufficiently close to $p, J_{U}^{-}\left[q_{2}\right]$ is close to $J_{U}^{-}[p]$, so is in the interior of $K$. Now, $J_{U}^{+}\left[q_{1}\right] \cap J_{U}^{-}\left[q_{2}\right]$ is closed in $J_{U}^{+}\left[q_{1}\right] \cap K$ hence compact. In conclusion, 
$I_{U}^{+}\left[q_{1}\right] \cap I_{U}^{-}\left[q_{2}\right]$ is an open neighbourhood of $p$ which is convex, and such that for any $r, s \in I_{U}^{+}\left[q_{1}\right] \cap I_{U}^{-}\left[q_{2}\right]$ with $r \ll_{U} s$, the set $J_{U}^{+}[r] \cap J_{U}^{-}[s]$ is compact.

Definition 1. Such a neighbourhood $I_{U}^{+}\left[q_{1}\right] \cap I_{U}^{-}\left[q_{2}\right]$ is said to be convex globally hyperbolic. Any point of $M$ admits a convex globally hyperbolic neighbourhood.

We will use the next lemma several times in the article:

Lemma 2. Each point $x_{0}$ of $M$ admits a neighbourhood $U$ with the following properties:

(i) $U$ is convex globally hyperbolic.

(ii) There is on $U$ a smooth time function $T: U \rightarrow]-A, A[$, namely a smooth submersion $T$ whose fibers are smooth spacelike hypersurfaces of $U$, and such that $T$ increases along any future oriented causal curve in $U$.

(iii) In $U$, there is no triangle whose edges are lightlike geodesic segments.

Proof. A time function as in (ii) exists in a neighbourhood of 0 in $T_{x_{0}} M$. It is sufficient to push it on $M$ via the exponential map (of any metric in the conformal class of $g$ ), and to restrict it to $U$. Point (iii), namely the non existence of local lightlike geodesic triangles, is a standard fact of causality on Lorentz manifolds (see [BE]).

\subsection{The zero set of a causal conformal vector field}

The structure of the zero set of a general Lorentzian conformal vector field is not easy to understand. Nevertheless, in the case of a causal conformal vector field, we know locally how this set looks like. What follows is a slight improvement of results obtained by F.Leitner in [L] (Theorem 1, p. 11).

Proposition 2. Let $Z(X)$ be the zero set of a causal conformal vector field $X$ on a Lorentz manifold $(M, g)$. Then, for every $p \in Z(X)$, there is an open set $U$ containing $p$, such that either $U \cap Z(X)=\{p\}$ or $U \cap Z(X)=[\gamma]$, where $[\gamma]$ is a lightlike geodesic segment.

Proof. Let us call $\phi_{X}^{t}$ the (local) flow associated to $X$. The first observation is:

Lemma 3. If $x \in Z(X)$ then there exists $\lambda \in \mathbf{R}$ such that $D_{x} \phi_{X}^{t}=e^{\lambda t} I d$. In particular, any nontrivial causal conformal vector field which vanishes is essential.

Proof. For all $t$ for which $\phi_{X}^{t}$ is defined, $D_{x} \phi_{X}^{t}$ is a conformal linear transformation of $T_{x} M$. Thus, it is of the form $e^{\lambda t} A(t)$, with $A(t) \in O(1, n-1)$ and $A\left(t t^{\prime}\right)=A(t) A\left(t^{\prime}\right)$ for $t, t^{\prime}$ sufficiently small. If $t \mapsto A(t)$ is nontrivial, then given $U$ a convex neighbourhood of $x$, and $y \in C_{U}^{+}[x], t \mapsto \phi_{X}^{t} . y$ is a curve of $C_{U}^{+}[x]$, transverse to any lightlike geodesic segment of $C_{U}^{+}[x]$ it 
meets. So the curve has spacelike tangent vectors, contradicting the causal nature of $X$.

The last assertion of the lemma is clear when $\lambda \neq 0$. Now, if $\lambda=0$, $D_{x} \phi_{X}^{t}=I d$. But since a Lorentzian isometry fixing a point is conjugated, around this point, to its differential, we see that if $X$ is inessential, it has to be trivial.

We now use the conclusions of [L], Theorem 1, p.11. Let $X$ be a conformal vector field on $(M, g)$, such that at any $x \in Z(X), D_{x} \phi_{X}^{t}=I d$. Then if $x_{0} \in Z(X)$, there is a neighbourhood $U$ of $x_{0}$, and a lightlike geodesic $[\gamma] \subset U$ (possibly reduced to a point) such that $Z(X) \cap U \subset[\gamma]$.

In fact, our conformal vector field $X$ does not exactly satisfy the asumptions of the theorem of Leitner, since we just proved that if $x \in Z(X)$, $D_{x} \phi_{X}^{t}=e^{\lambda t} I d$. But the proof of Leitner works without any change, even under this weaker hypothesis on $X$.

Now, let $x \in Z(X)$. If $x$ is isolated, we are in the case $(i)$ of Proposition 2 . If not, we get, by $[\mathrm{L}]$ the existence of a neighbourhood $U$, and a non trivial lightlike geodesic $[\gamma] \subset U$ such that $Z(X) \cap U \subset[\gamma]$, as well as a sequence $\left(x_{i}\right)_{i \in \mathbb{N}}$ of $Z(X) \cap[\gamma]$ converging to $x$. Shrinking $U$ and $[\gamma]$ if necessary, we can choose a conformal parametrization $u \mapsto \gamma(u)$ of $[\gamma]$, defined on [0,1]. If $\phi_{X}^{t}$ is the local flow associated to $X$, then $\phi_{X}^{t}(\gamma(u))=\gamma\left(\tau_{t}(u)\right)$, for a family of homographic transformations $\tau_{t}$. But since $\phi_{X}^{t}$ fixes every $x_{i}=\gamma\left(u_{i}\right)$, $\tau_{t}\left(u_{i}\right)=u_{i}$ for all $i \in \mathbb{N}$, what implies that each $\tau_{t}$ is in fact the identity. So, for any $u \in[0,1], \phi_{X}^{t}(\gamma(u))=\gamma(u)$, what proves that $[\gamma] \subset Z(X)$. Shrinking $U$ if necessary, we get $Z(X) \cap U=[\gamma] \cap U$, and we are in the second case of the proposition.

As an easy consequence of Proposition 2, we get the:

Corollary 3. If $(M, g)$ is a compact Lorentz manifold, and if $X$ is a causal conformal vector field on $M$, then $Z(X)$ is a finite union of points and closed lightlike geodesics.

\subsection{The Cotton and Weyl tensors}

Let $(M, g)$ be a smooth Lorentz manifold of dimension greater or equal to three. From the conformal point of view, two interesting tensors can be built.

The Weyl tensor is given by the formula:

$$
\tilde{W}=R-\frac{S}{2 n(n-1)} g \cdot g-\frac{1}{n-2}\left(R i c-\frac{S}{n} g\right) \cdot g
$$

here, $R, R i c$ and $S$ stand for the Riemann, Ricci and Scalar curvature associated to $g$, and $h . q$ stands for the Kulkarni-Nomizu product of two symetric 2 -tensors (see [B] p.47). 
The Weyl tensor is always zero in dimension 3 , and in dimension $\geq 4$, the vanishing of $\tilde{W}$ is a necessary and sufficient condition for the metric $g$ to be conformally flat. We will denote by $W$ the $(1,3)$ tensor defined by $g(W(X, Y, Z), T)=\tilde{W}(X, Y, Z, T)$. For any (local) conformal diffeomorphism $\phi$ of $(M, g), D \phi(W(X, Y, Z))=W(D \phi(X), D \phi(Y), D \phi(Z))$.

The Schouten tensor on $(M, g)$ is given by:

$$
S=\frac{1}{n-2}\left(R i c-\frac{S}{2(n-1)} g\right)
$$

Then, one defines the Cotton tensor by $C(X, Y, Z)=\left(\nabla_{X} S\right)(Y, Z)-$ $\left(\nabla_{Y} S\right)(X, Z)$.

In any dimension, the Cotton tensor vanishes as soon as $g$ is conformally flat (see [E] p.91). In dimension 3, the vanishing of $C$ is a necessary and sufficient condition for the metric $g$ to be conformally flat. Notice also that in dimension three, the Cotton tensor is conformally invariant, so that for any (local) conformal diffeomorphism $\phi, C(X, Y, Z)=C(D \phi(X), D \phi(Y), D \phi(Z))$.

\section{Some general dynamical aspects}

\subsection{Cartan decomposition for the derivative cocycle}

In all this section, $\left(\phi_{k}\right)$ denotes a sequence of conformal diffeomorphisms of a Lorentz manifold $(M, g)$. This means that for all $k \in \mathbb{N},\left(\phi_{k}\right)^{*} g=e^{2 \sigma_{k}} g$, for $\sigma_{k} \in C^{\infty}(M)$. We suppose that there is $x_{0} \in M$ such that $x_{k}=\phi_{k}\left(x_{0}\right)$ has a limit point $x_{\infty} \in M$. Let us choose a smooth frame field $x \mapsto$ $\left(E_{1}(x), E_{2}(x), \ldots, E_{n}(x)\right)$ (resp. $\left.y \mapsto\left(F_{1}(y), F_{2}(y), \ldots, F_{n}(y)\right)\right)$ in a neighbourhood of $x_{0}$ (resp. of $\left.x_{\infty}\right)$. We suppose moreover that $\left(E_{1}(x), \ldots, E_{n}(x)\right.$ ) and $\left(F_{1}(y), \ldots, F_{n}(y)\right)$ satisfy $g_{x}\left(E_{1}(x), E_{2}(x)\right)=1\left(\operatorname{resp} . g_{y}\left(F_{1}(y), F_{2}(y)\right)=\right.$ 1 ) and $g_{x}\left(E_{i}(x), E_{i}(x)\right)=1, i \geq 3\left(\operatorname{resp} . \quad g_{y}\left(F_{i}(y), F_{i}(y)\right)=1, i \geq 3\right.$ ), all the other products being zero. The frame $\left(E_{1}\left(x_{0}\right), \ldots, E_{n}\left(x_{0}\right)\right)$ (resp. $\left.\left(F_{1}\left(\phi_{k}\left(x_{0}\right)\right), \ldots, F_{n}\left(\phi_{k}\left(x_{0}\right)\right)\right)\right)$ yields an identification of $T_{x_{0}} M\left(\operatorname{resp} . T_{\phi_{k}\left(x_{0}\right)} M\right)$ with Minkowski's space $\mathbf{R}^{1, n-1}$. Under this identification, the differential $D_{x_{0}} \phi_{k}$ is a matrix $M_{k}\left(x_{0}\right)$ in $\mathbf{R} \times O(1, n-1)$. The projection on the $\mathbf{R}$ factor is just the square root of the conformal distorsion, namely $e^{\sigma_{k}\left(x_{0}\right)}$. Recall that there is a Cartan decomposition $O(1, n-1)=K A K$, where $K$ is the maximal compact subgroup of $O(1, n-1)$, and $A$ is a maximal abelian subgroup in $O(1, n-1)$. We perform a Cartan decomposition of the sequence $M_{k}\left(x_{0}\right)$, so that $M_{k}\left(x_{0}\right)$ writes as a product $L_{1}^{(k)}\left(x_{0}\right) D_{k}\left(x_{0}\right) L_{2}^{(k)}\left(x_{0}\right)$. The two matrix $L_{1}^{(k)}\left(x_{0}\right)$ and $L_{2}^{(k)}\left(x_{0}\right)$ are in $K$ and $D_{k}\left(x_{0}\right)$ is a diagonal matrix 
of the form

$$
e^{\sigma_{k}\left(x_{0}\right)}\left(\begin{array}{ccccc}
e^{\lambda_{k}\left(x_{0}\right)} & & & & \\
& e^{-\lambda_{k}\left(x_{0}\right)} & & & \\
& & 1 & & \\
& & & \ddots & \\
& & & & 1
\end{array}\right)
$$

with $\lambda_{k}\left(x_{0}\right) \geq 0$

In what follows, we will use the notation $\delta_{k}^{+}\left(x_{0}\right)=\sigma_{k}\left(x_{0}\right)+\lambda_{k}\left(x_{0}\right)$ and $\delta_{k}^{-}\left(x_{0}\right)=\sigma_{k}\left(x_{0}\right)-\lambda_{k}\left(x_{0}\right)$. Remark that one always has $\delta_{k}^{-} \leq \sigma_{k} \leq \delta_{k}^{+}$.

Definition 2. A sequence $\left(\phi_{k}\right)$ such that $x_{k}=\phi_{k}\left(x_{0}\right)$ tends to $x_{\infty}$ is said to be simple if:

$(i) e^{\lambda_{k}\left(x_{0}\right)}, e^{\sigma_{k}\left(x_{0}\right)}, e^{\delta_{k}^{+}\left(x_{0}\right)}$ and $e^{\delta_{k}^{-}\left(x_{0}\right)}$ all have a limit in $\mathbf{R} \cup\{+\infty\}$ when $k \rightarrow+\infty$.

(ii) The two sequences $L_{1}^{(k)}\left(x_{0}\right)$ and $L_{2}^{(k)}\left(x_{0}\right)$ converge in $K$.

Now, if $\left(\phi_{k}\right)$ is a simple sequence of conformal transformations such that $\phi_{k}\left(x_{0}\right) \rightarrow x_{\infty}$, then there is a sequence $\left(X_{1}^{(k)}, X_{2}^{(k)}, \ldots, X_{n}^{(k)}\right)$ of frames on $T_{x_{0}} M$, and a sequence $\left(Y_{1}^{(k)}, Y_{2}^{(k)}, \ldots, Y_{n}^{(k)}\right)$ of frames on $T_{x_{k}} M$ such that:

(i) $\left(X_{1}^{(k)}, X_{2}^{(k)}, \ldots, X_{n}^{(k)}\right)$ tends to a frame $\left(X_{1}, X_{2}, \ldots, X_{n}\right)$ of $T_{x_{0}} M$.

(ii) $\left(Y_{1}^{(k)}, Y_{2}^{(k)}, \ldots, Y_{n}^{(k)}\right)$ tends to a frame $\left(Y_{1}, Y_{2}, \ldots, Y_{n}\right)$ of $T_{x_{\infty}} M$.

(iii) $D_{x_{0}} \phi_{k}\left(X_{1}^{(k)}\right)=e^{\delta_{k}^{+}\left(x_{0}\right)} Y_{1}^{(k)}$

$$
\begin{aligned}
& D_{x} \phi^{t_{k}}\left(X_{2}^{(k)}\right)=e^{\delta_{k}^{-}\left(x_{0}\right)} Y_{2}^{(k)} \\
& D_{x} \phi^{t_{k}}\left(X_{i}^{(k)}\right)=e^{\sigma_{k}\left(x_{0}\right)} Y_{i}^{(k)} \text { for } i \geq 3,
\end{aligned}
$$

Indeed, we just define $X_{1}^{(k)}, X_{2}^{(k)}, \ldots, X_{n}^{(k)}$ as $\left(L_{2}^{(k)}\left(x_{0}\right)\right)^{-1}\left(E_{1}\left(x_{0}\right), \ldots, E_{n}\left(x_{0}\right)\right)$, and $Y_{1}^{(k)}, Y_{2}^{(k)}, \ldots, Y_{n}^{(k)}$ as $L_{1}^{(k)}\left(x_{0}\right)\left(F_{1}\left(x_{k}\right), \ldots, F_{n}\left(x_{k}\right)\right)$.

Notice that for all $k \in \mathbb{N} \cup\{\infty\}, X_{1}^{(k)}, X_{2}^{(k)}, Y_{1}^{(k)}$ and $Y_{2}^{(k)}$ are isotropic.

\subsection{Stability and dynamical normality for conformal trans- formations}

We now recall some usefull dynamical notions, first introduced by Zeghib in $[\mathrm{Z}]$.

Definition 3. Let $\left(\phi_{k}\right)$ be a simple sequence of conformal transformations of $(M, g)$, such that $\phi_{k}\left(x_{0}\right) \rightarrow x_{\infty}$. The stable space at $x_{0}$ for the sequence $\left(\phi_{k}\right)$ is defined as the subspace $H_{x_{0}}^{<}=\left\{u \in T_{x_{0}} M \mid \exists\left(u_{k}\right) \subset T_{x_{0}} M, u_{k} \rightarrow\right.$ $u$, and $D_{x_{0}} \phi_{k}\left(u_{k}\right)$ is bounded $\}$. We also define the strongly stable space at $x_{0}$ as $H_{x_{0}}^{<<}=\left\{u \in T_{x_{0}} M \mid \exists\left(u_{k}\right) \subset T_{x_{0}} M, u_{k} \rightarrow u\right.$, and $D_{x_{0}} \phi_{k}\left(u_{k}\right) \rightarrow 0 \in$ $\left.T_{x_{\infty}} M\right\}$. 
The sequence $\left(\phi_{k}\right)$ is said to be stable at $x_{0}$ if $H_{x_{0}}^{<}=T_{x_{0}} M$, and strongly stable when $H_{x_{0}}^{<<}=T_{x_{0}} M$.

The following lemma describes the possible stable and strongly stable subspaces for a sequence of Lorentzian conformal transformations.

Lemma 4. Let $\left(\phi_{k}\right)$ be a simple sequence of conformal transformations of a Lorentz manifold $(M, g)$, such that $x_{k}=\phi_{k}\left(x_{0}\right)$ tends to $x_{\infty}$. Then:

1) The sequence $\left(\phi_{k}\right)$ is strongly stable at $x_{0}$ if and only if $\lim _{k \rightarrow+\infty}\left(e^{\delta_{k}^{+}\left(x_{0}\right)}\right)=$ 0 .

2) The sequence $\left(\phi_{k}\right)$ is stable at $x_{0}$ without being strongly stable if and only if $\left(e^{\delta_{k}^{+}\left(x_{0}\right)}\right)$ tends to $e^{\delta_{\infty}^{+}}$, for $\delta_{\infty}^{+} \in \mathbf{R}$. Two subcases can then occur:

(i) $D_{x_{0}} \phi_{k}$ is bounded. In this case $H_{x_{0}}^{<<}=\{0\}$.

(ii) $D_{x_{0}} \phi_{k}$ is not bounded. The strongly stable space $H_{x_{0}}^{<<}$is a lightlike hyperplane. With the notations above $H_{x_{0}}^{<<}=\left(X_{2}\right)^{\perp}$ (the g-orthogonal of $\left.X_{2}\right)$.

3) When the sequence $\left(e^{\delta_{k}^{+}\left(x_{0}\right)}\right)$ tends to $+\infty$, the sequence $\left(\phi_{k}\right)$ is not stable at $x_{0}$. To be more precise, the two following subscases can occur:

(i) The sequence $e^{\sigma_{k}\left(x_{0}\right)}$ tends to $e^{\sigma_{\infty}}$, for $\sigma_{\infty} \in \mathbf{R}$. The stable space $H_{x_{0}}^{<}$is the lightlike hyperplane $\left(X_{2}\right)^{\perp}$. The strongly stable space $H_{x_{0}}^{<<}$is the lightlike line $\mathbf{R} \cdot X_{2}$.

(ii) The sequence $e^{\sigma_{k}\left(x_{0}\right)}$ tends to $+\infty$. Then, if $\lim _{k \rightarrow+\infty} \delta_{k}^{-} \neq+\infty$, then $H_{x_{0}}^{<}=H_{x_{0}}^{<<}=\mathbf{R} \cdot X_{2}$. If $\lim _{k \rightarrow+\infty} \delta_{k}^{-}=+\infty$, then $H_{x_{0}}^{<}=H_{x_{0}}^{<<}=\{0\}$.

In what follows, we will mainly be concerned with the two first cases of the lemma.

Let us now introduce another dynamical notion, namely that of dynamical normality.

Definition 4. Let $x_{0}$ be a point of a manifold $M$, and $\left(\phi_{k}\right)$ be a sequence of homeomorphisms $M$ such that $\lim _{k \rightarrow+\infty} \phi_{k}\left(x_{0}\right)=x_{\infty}$ (we put $x_{\infty}=\infty$ if the sequence $\phi_{k}\left(x_{0}\right)$ leaves every compact subset of $\left.M\right)$. We define the set:

$$
\mathcal{D}_{\left(\phi_{k}\right)}\left(x_{0}\right)=\bigcup_{x_{k} \rightarrow x_{0}}\left\{\text { accumulation points of }\left(\phi_{k}\left(x_{k}\right)\right)\right\}
$$

The union is taken over all sequences converging to $x_{0}$.

We say that the point $x_{0}$ is dynamically normal (for the sequence $\phi_{k}$ ), or equivalently that $\left(\phi_{k}\right)$ is dynamically normal at $x_{0}$, iff $\mathcal{D}_{\left(\phi_{k}\right)}\left(x_{0}\right)=\left\{p_{\infty}\right\}$.

For Lorentzian conformal transformations, the link between dynamical normality and stability is made clear by the following proposition: 
Proposition 3. Let $\left(\phi_{k}\right)$ be a sequence of conformal diffeomorphisms of a Lorentz manifold $(M, g)$, and let $x_{0} \in M$ such that $\phi_{k}\left(x_{0}\right) \rightarrow x_{\infty}$. If $\left(\phi_{k}\right)$ is dynamically normal at $x_{0}$, then $\left(\phi_{k}\right)$ is stable at $x_{0}$.

Notice that the converse of the proposition is false. For example, let us consider the space $\mathbf{E i n}_{n}$, namely the conformal compactification of $n$ dimensional Minkowski's space. We will study this space in section 6, and see that any translation flow on Minkowski's space extends to a conformal flow on $\mathbf{E i n}_{n}$. In particular, these flows have fixed points on $\mathbf{E i n}_{n}$, and the differential at these fixed points are always identity. Nevertheless, the fixed points are never dynamically normals. So, stability and dynamical normality are really distinct notions for Lorentzian conformal transformations.

Proof. Suppose on the contrary that $\left(\phi_{k}\right)$ is not stable at $x_{0}$, and choose a sequence $\left(v_{k}\right) \subset T_{x_{0}} M$, such that $\left(v_{k}\right)$ tends to some non zero vector $v_{\infty}$, and $D_{x_{0}}\left(\phi_{k}\right)\left(v_{k}\right)$ is unbounded. Looking at a subsequence, we suppose that $\left\|D_{x_{0}}\left(\phi_{k}\right)\left(v_{k}\right)\right\|$ tends to $+\infty$ (the norm being taken for any Riemannian metric on a relatively compact neighbourhood of $\left.x_{\infty}\right)$. We suppose moreover that $\left(v_{k}\right)$ is a sequence of lightlike vectors (which is always possible). The lightlike vectors $v_{k}$ (for $k \in \mathbb{N} \cup\{\infty\}$ ) are tangent to a family $\left[\gamma_{k}\right]$ of lightlike geodesics. Let $U$ be a small neighbourhood around $x_{0}$. By $\left[\gamma_{k}\right]_{U}$, we will denote the connected component of $\left[\gamma_{k}\right] \cap U$ which contains $x_{0}$. We choose conformal parametrizations $\gamma_{k}:[0,1] \rightarrow M$ of $\left[\gamma_{k}\right]_{U}$, such that $\left(\gamma_{k}\right)$ converges for the $C^{1}$ topology to a conformal parametrization $\gamma_{\infty}$ of the geodesic $\left[\gamma_{\infty}\right]_{U}$ passing through $x_{0}$ with tangent vector $v_{\infty}$. By an affine change of parameter (which still yields conformal parametrizations), and shrinking the interval of definition, we suppose moreover that $\gamma_{k}^{\prime}(0)=v_{k}$, $k \in \mathbb{N} \cup\{\infty\}$. Taking a subsequence of $\left(\phi_{k}\right)$ if necessary, we can suppose that the vectors $D_{x_{0}}\left(v_{k}\right)$ converge projectively to the direction supported by some vector $v_{\infty}^{\prime} \in T_{x_{\infty}} M$. Any lightlike direction $D_{x_{0}}\left(v_{k}\right)$ defines a lightlike conformal geodesic $\left[\alpha_{k}\right]$, and $v_{\infty}$ defines $\left[\alpha_{\infty}\right]$ as well. We choose a small neighbourhood $U^{\prime}$ of $x_{\infty}$ and conformal parametrizations $\alpha_{k}:[0,1] \rightarrow M$, $\alpha_{\infty}:[0,1] \rightarrow M$, of $\left[\alpha_{k}\right] \cap U^{\prime}$ and $\left[\alpha_{\infty}\right] \cap U^{\prime}$ respectively, such that $\alpha_{k}$ $C^{1}$-converges to $\alpha_{\infty}$. Now, because conformal transformations respect conformal parametrizations, $\phi_{k} \circ \gamma_{k}$ is a conformal parametrization of a segment of the lightlike geodesic $\left[\alpha_{k}\right]$, starting at $x_{k}$. Hence, there is an homography $h_{k}$ such that $\phi_{k} \circ \gamma_{k}(t)=\alpha_{k} \circ h_{k}(t)$, fot $t \in[0,1]$. Derivating at $t=0$, we get $D_{x_{0}} \phi_{k}\left(v_{k}\right)=h_{k}^{\prime}(0) \cdot \alpha_{k}^{\prime}(0)$. But $\alpha_{k}^{\prime}(0)$ is a bounded (even converging) sequence of vectors, so that $\left|h_{k}^{\prime}(0)\right|$ tends to $+\infty$. We conclude that $\left(h_{k}\right)$ is sequence of projective transformations fixing 0 , which tends to infinity. Such sequences can be dynamically normal at 0 only if their derivatives at 0 tend to 0 . We infer that $\left(h_{k}\right)$ is not dynamically normal at 0 . More precisely, there is a sequence $\left(t_{k}\right)$ tending to 0 , such that $h_{k}\left(t_{k}\right)$ accumulates at $\frac{1}{2}$. Thus, $\phi_{k}\left(\gamma_{k}\left(t_{k}\right)\right)$ accumulates at $\alpha_{\infty}\left(\frac{1}{2}\right)$, what proves that $\left(\phi_{k}\right)$ is not dynamically normal at $x_{0}$. 


\subsection{Effects of the dynamics on the Weyl and Cotton tensors}

Proposition 4. Let $\left(\phi_{k}\right)$ be a sequence of conformal transformations of a Lorentz manifold $(M, g)$, whose dimension is greater or equal to four. We suppose that $\lim _{k \rightarrow+\infty} \phi_{k}\left(x_{0}\right)=x_{\infty}$ for some $x_{\infty} \in M$. We suppose also that $\left(\phi_{k}\right)$ is stable at $x_{0}$. Then, denoting by $W$ the Weyl tensor of the conformal structure on $M$ :

(i) If $W_{x_{\infty}}=0$, then $W_{x_{0}}=0$.

(ii) If $D_{x_{0}} \phi_{k}$ is unbounded, then $\operatorname{Im} W_{x_{0}} \subset H_{x_{0}}^{<<}$(here $\operatorname{Im} W_{x_{0}}$ denotes the set of all possible values of the $(1,3)$ tensor $W$ at $\left.x_{0}\right)$.

(iii) If $\left(\phi_{k}\right)$ is strongly stable at $x_{0}$, then $W_{x_{0}}=0$.

Proof. Let us precise some notations before begining the proof.

We will note $x_{k}=\phi_{k}\left(x_{0}\right)$. For $i, j$ and $l$ in $\{1, \ldots, n\}$, we will also use the notation $W_{x_{0}}(i, j, l)$ to denote the set of values $W\left(X_{\sigma(i)}, X_{\sigma(j)}, X_{\sigma(l)}\right)$, when $\sigma$ describes the set of all permutations of $\{i, j, l\}$.

Let us also recall that with the notations of section 3.1 , when $\left(\phi_{k}\right)$ is stable at $x_{0}$ but not strongly stable, the strongly stable subspace $H_{x_{0}}^{<<}$is the subspace spanned by $X_{2}, X_{3}, \ldots, X_{n}$ (see lemma 4 ).

We begin by proving $(i)$ in the case where $D_{x_{0}} \phi_{k}$ is bounded. Suppose that we have $W_{x_{\infty}}=0$ but $W_{x_{0}} \neq 0$. We then choose $X, Y, Z$ in $T_{x_{0}} M$ such that $W_{x_{0}}(X, Y, Z) \neq 0$.

Now $D_{x_{0}} \phi_{k}\left(W_{x_{0}}(X, Y, Z)\right)=W_{x_{k}}\left(D_{x_{0}} \phi_{k}(X), D_{x_{0}} \phi_{k}(Y), D_{x_{0}} \phi_{k}(Z)\right)$. Since $D_{x_{0}} \phi_{k}$ is bounded, we can suppose, looking at a subsequence of $\left(\phi_{k}\right)$ if necessary, that $D_{x_{0}} \phi_{k}(X), D_{x_{0}} \phi_{k}(Y), D_{x_{0}} \phi_{k}(Z)$ and $D_{x_{0}} \phi_{k}\left(W_{x_{0}}(X, Y, Z)\right)$ converge to $X_{\infty}, Y_{\infty}, Z_{\infty}$ and $W_{\infty}$ in $T_{x_{\infty}} M$. Again because $D_{x_{0}} \phi_{k}$ is bounded, we are sure that $W_{\infty} \neq 0$, since $W_{x_{0}}(X, Y, Z) \neq 0$.

We thus get $W_{x_{\infty}}\left(X_{\infty}, Y_{\infty}, Z_{\infty}\right)=W_{\infty}$, a contradiction with the hypothesis $W_{x_{\infty}}=0$.

We will now prove $(i),(i i)$ and (iii) under the hypothesis that $D_{x_{0}} \phi_{k}$ is simple, stable and unbounded. This imply in particular $\lim _{k \rightarrow+\infty} \sigma_{k}=-\infty$ and $\delta_{k}^{+}$bounded. We will often use this implicitely in the rest of the proof.

Lemma 5. Let $v_{k} \in T_{x_{0}} M$ be a sequence of vectors converging to $v_{\infty} \in$ $T_{x_{0}} M$. Then,

(i) if $\lim _{k \rightarrow+\infty}\left(e^{-\delta_{k}^{+}\left(x_{0}\right)} D_{x_{0}} \phi_{k}\left(v_{k}\right)\right)=0$, then $v_{\infty} \in H_{x_{0}}^{<<}$.

(ii) if $\lim _{k \rightarrow+\infty}\left(e^{-\sigma_{k}\left(x_{0}\right)} D_{x_{0}} \phi_{k}\left(v_{k}\right)\right)=0$, then $v_{\infty}=\mu X_{2}, \mu \in \mathbf{R}$.

(iii) if $\lim _{k \rightarrow+\infty}\left(e^{-\delta_{k}^{-}\left(x_{0}\right)} D_{x_{0}} \phi_{k}\left(v_{k}\right)\right)=0$, then $v_{\infty}=0$.

Let $(i, j, l)$ three indices in $\{1, \ldots, n\}$. The conformal invariance of the Weyl tensor yields for all $k \in \mathbb{N}$ :

$W_{x_{k}}\left(D_{x_{0}} \phi_{k}\left(X_{i}^{(k)}\right), D_{x_{0}} \phi_{k}\left(X_{j}^{(k)}\right), D_{x_{0}} \phi_{k}\left(X_{l}^{(k)}\right)\right)=D_{x_{0}} \phi_{k}\left(W_{x_{0}}\left(X_{i}^{(k)}, X_{j}^{(k)}, X_{l}^{(k)}\right)\right)$ 
We can now prove:

- $W(1,1,2)=\{0\}$.

We compute :

$e^{-\sigma_{k}\left(x_{0}\right)} D_{x_{0}} \phi_{k}\left(W_{x_{0}}\left(X_{1}^{(k)}, X_{2}^{(k)}, X_{1}^{(k)}\right)\right)=e^{2 \delta_{k}^{+}\left(x_{0}\right)+\delta_{k}^{-}\left(x_{0}\right)-\sigma_{k}\left(x_{0}\right)} W_{x_{k}}\left(Y_{1}^{(k)}, Y_{2}^{(k)}, Y_{1}^{(k)}\right)$

$$
=e^{2 \sigma_{k}\left(x_{0}\right)+\lambda_{k}\left(x_{0}\right)} W_{x_{k}}\left(Y_{1}^{(k)}, Y_{2}^{(k)}, Y_{1}^{(k)}\right) .
$$

This latter term tends to zero since in both stable and strongly stable case, $\lim _{k \rightarrow+\infty}\left(2 \sigma_{k}\left(x_{0}\right)+\lambda_{k}\left(x_{0}\right)\right)=-\infty$. Lemma 5 then ensures that $W_{x_{0}}\left(X_{1}, X_{2}, X_{1}\right)=\mu X_{2}$. If $\mu \neq 0$, then looking at the $(0,4)$-Weyl tensor instead of the $(1,3)$, we get $W_{x_{0}}\left(X_{1}, X_{2}, X_{1}, X_{1}\right) \neq 0$, which is not possible, because of Bianchi's identities. We thus get $W_{x_{0}}\left(X_{1}, X_{2}, X_{1}\right)=0$. The same conclusion holds, with the same proof for $W_{x_{0}}(U, V, W)$, when two of these three vectors are equal to $X_{1}$, and the other to $X_{2}$.

- $W_{x_{0}}(1,2,2)=\{0\}$.

$e^{-\delta_{k}^{-}\left(x_{0}\right)} D_{x_{0}} \phi_{k}\left(W_{x_{0}}\left(X_{1}^{(k)}, X_{2}^{(k)}, X_{2}^{(k)}\right)\right)=e^{2 \sigma_{k}\left(x_{0}\right)} W_{x_{k}}\left(Y_{1}^{(k)}, Y_{2}^{(k)}, Y_{2}^{(k)}\right)$. Since the last term tends to zero, lemma 5 implies $W_{x_{0}}\left(X_{1}, X_{2}, X_{2}\right)=0$. The same conclusion holds for $W_{x_{0}}(U, V, W)$, when two of these three vectors are equal to $X_{2}$, and the other to $X_{1}$.

- $W_{x_{0}}(2,2, i)=\{0\}$, for $i \geq 3$.

$e^{-\delta_{k}^{-}\left(x_{0}\right)} D_{x_{0}} \phi_{k}\left(W_{x_{0}}\left(X_{2}^{(k)}, X_{i}^{(k)}, X_{2}^{(k)}\right)\right)=e^{\delta_{k}^{-}\left(x_{0}\right)+\sigma_{k}\left(x_{0}\right)} W_{x_{k}}\left(Y_{1}^{(k)}, Y_{2}^{(k)}, Y_{2}^{(k)}\right)$.

This last term clearly tends to zero, what gives the conclusion by lemma 5 .

- $W_{x_{0}}(2, i, j)=\{0\}$, for $i, j \geq 3$.

$e^{-\delta_{k}^{-}\left(x_{0}\right)} D_{x_{0}} \phi_{k}\left(W_{x_{0}}\left(X_{2}^{(k)}, X_{i}^{(k)}, X_{j}^{(k)}\right)\right)=e^{2 \sigma_{k}\left(x_{0}\right)} W_{x_{k}}\left(Y_{2}^{(k)}, Y_{i}^{(k)}, Y_{j}^{(k)}\right)$. This last term tends to zero and we conclude by lemma 5 .

- For $i \geq 3, W_{x_{0}}(1,2, i)=\{0\}$.

$e^{-\delta_{k}^{-}\left(x_{0}\right)} D_{x_{0}} \phi_{k}\left(W_{x_{0}}\left(X_{1}^{(k)}, X_{2}^{(k)}, X_{i}^{(k)}\right)\right)=e^{\delta_{k}^{+}\left(x_{0}\right)+\sigma_{k}\left(x_{0}\right)} W_{x_{k}}\left(Y_{2}^{(k)}, Y_{i}^{(k)}, Y_{j}^{(k)}\right)$. Under the asumption of stability (or strong stability), $\lim _{k \rightarrow+\infty}\left(\delta_{k}^{+}\left(x_{0}\right)+\right.$ $\left.\sigma_{k}\left(x_{0}\right)\right)=-\infty$. So, the last term tends to zero and we conclude by lemma 5 .

- For $i \geq 3, W_{x_{0}}(1,1, i)=\{0\}$ in case $(i i i)$ and $(i)$, and $W_{x_{0}}(1,1, i) \in H_{x_{0}}^{<<}$ in case $(i i)$.

$e^{-\sigma_{k}\left(x_{0}\right)} D_{x_{0}} \phi_{k}\left(W_{x_{0}}\left(X_{1}^{(k)}, X_{i}^{(k)}, X_{1}^{(k)}\right)\right)=e^{2 \delta_{k}^{+}\left(x_{0}\right)} W_{x_{k}}\left(Y_{1}^{(k)}, Y_{i}^{(k)}, Y_{1}^{(k)}\right)$. If we are in the case $\left(\right.$ iii), $\lim _{k \rightarrow+\infty} \delta_{k}^{+}=-\infty$, and we conclude that the last term tends to zero. In the case $(i), e^{2 \delta_{k}^{+}\left(x_{0}\right)}$ is bounded, but by hypothesis $\lim _{k \rightarrow+\infty} W_{x_{k}}\left(Y_{1}^{(k)}, Y_{i}^{(k)}, Y_{1}^{(k)}\right)=0$. So, in both of these cases, we get $W_{x_{0}}\left(X_{1}, X_{i}, X_{1}\right)=\mu X_{2}, \mu \in \mathbf{R}$. But once again, by Bianchi identity, $g_{x_{0}}\left(X_{1}, W_{x_{0}}\left(X_{1}, X_{i}, X_{1}\right)\right)=0$, so that $\mu=0$, and we are done. 
When we are in case $(i i)$ :

$e^{-\delta_{k}^{+}\left(x_{0}\right)} D_{x_{0}} \phi_{k}\left(W_{x_{0}}\left(X_{1}^{(k)}, X_{i}^{(k)}, X_{1}^{(k)}\right)\right)=e^{\delta_{k}^{+}\left(x_{0}\right)+\sigma_{k}\left(x_{0}\right)} W_{x_{k}}\left(Y_{1}^{(k)}, Y_{i}^{(k)}, Y_{1}^{(k)}\right)$

which tends to zero, and lemma 5 gives the conclusion.

- For $i, j \geq 3, W_{x_{0}}(1, i, j)=\{0\}$ in case $(i i i)$ and $(i)$, and $W_{x_{0}}(1, i, j) \in \mathbf{R} \cdot X_{2}$ in case $(i i)$.

$$
\begin{aligned}
e^{-\delta_{k}^{-}\left(x_{0}\right)} D_{x_{0}} \phi_{k}\left(W_{x_{0}}\left(X_{1}^{(k)}, X_{i}^{(k)}, X_{j}^{(k)}\right)\right) & =e^{\delta_{k}^{+}\left(x_{0}\right)+2 \sigma_{k}\left(x_{0}\right)-\delta_{k}^{-}\left(x_{0}\right)} W_{x_{k}}\left(Y_{1}^{(k)}, Y_{i}^{(k)}, Y_{j}^{(k)}\right) \\
& =e^{2 \delta_{k}^{+}\left(x_{0}\right)} W_{x_{k}}\left(Y_{1}^{(k)}, Y_{i}^{(k)}, Y_{j}^{(k)}\right) .
\end{aligned}
$$

If we are in the case $(i i i), \lim _{k \rightarrow+\infty} \delta_{k}^{+}=-\infty$, and we conclude that the last term tends to zero. In the case $(i), e^{2 \delta_{k}^{+}\left(x_{0}\right)}$ is bounded, but by hypothesis $\lim _{k \rightarrow+\infty} W_{x_{k}}\left(Y_{1}^{(k)}, Y_{i}^{(k)}, Y_{j}^{(k)}\right)=0$. So, in both of these cases, we get $W_{x_{0}}\left(X_{1}, X_{i}, X_{j}\right)=0$, by lemma 5 .

When we are in case $(i i)$ :

$$
e^{-\sigma_{k}\left(x_{0}\right)} D_{x_{0}} \phi_{k}\left(W_{x_{0}}\left(X_{1}^{(k)}, X_{i}^{(k)}, X_{j}^{(k)}\right)\right)=e^{\delta_{k}^{+}\left(x_{0}\right)+\sigma_{k}\left(x_{0}\right)} W_{x_{k}}\left(Y_{1}^{(k)}, Y_{i}^{(k)}, Y_{j}^{(k)}\right)
$$

which tends to zero, and lemma 5 gives the conclusion.

- For $i, j, l \geq 3, W_{x_{0}}(i, j, l)=\{0\}$.

$e^{-\delta_{k}^{-}\left(x_{0}\right)} D_{x_{0}} \phi_{k}\left(W_{x_{0}}\left(X_{i}^{(k)}, X_{j}^{(k)}, X_{l}^{(k)}\right)\right)=e^{\delta_{k}^{+}\left(x_{0}\right)+\sigma_{k}\left(x_{0}\right)} W_{x_{k}}\left(Y_{i}^{(k)}, Y_{j}^{(k)}, Y_{l}^{(k)}\right)$

This last term tends to zero and we conclude thanks to lemma 5.

The proof of the following statement is much easier:

Proposition 5. Let $\left(\phi_{k}\right)$ be a sequence of conformal transformations of a three dimensional Lorentz manifold $(M, g)$. We suppose that $\lim _{k \rightarrow+\infty} \phi_{k}\left(x_{0}\right)=$ $x_{\infty}$ for some $x_{\infty} \in M$. We suppose also that $\left(\phi_{k}\right)$ is stable at $x_{0}$, and $D_{x_{0}} \phi_{k}$ is unbounded. Then the Cotton tensor $C$ vanishes at $x_{0}$.

Proof. Keeping the notations of the previous proposition, we get:

$C_{x_{0}}\left(X_{i}^{(k)}, X_{j}^{(k)}, X_{l}^{(k)}\right)=e^{\alpha_{k}\left(x_{0}\right)} C_{x_{k}}\left(Y_{i}^{(k)}, Y_{j}^{(k)}, Y_{l}^{(k)}\right)$, for all $k \in \mathbb{N}$, with $\lim _{k \rightarrow+\infty} \alpha_{k}=-\infty$, except in the case $i=j=l=1$. So, except when $(i, j, l)=(1,1,1), C_{x_{0}}\left(X_{i}^{(\infty)}, X_{j}^{(\infty)}, X_{l}^{(\infty)}\right)=0$, and since $C$ is antisymmetric in the two first variables, we also have $C_{x_{0}}\left(X_{1}^{(\infty)}, X_{1}^{(\infty)}, X_{1}^{(\infty)}\right)=0$, which finishes the proof. 


\section{Dynamics around a fixed point of a causal con- formal flow}

Let $X$ be a causal conformal vector field, and $x_{0}$ a point of $M$ such that $X\left(x_{0}\right)=0$. By proposition $2, x_{0}$ is either an isolated singularity of $X$, or lies on a lightlike geodesic of singular points of $X$.

\subsection{The case of an isolated singularity}

We first suppose that $X$ admits an isolated zero $x_{0} \in M$.

Proposition 6. There is an open set $U$ containing $x_{0}$ such that, changing $X$ into $-X$ if necessary, $\forall x \in I_{U}^{+}\left[x_{0}\right], \phi_{X}^{t}(x)$ is defined for all $t \in \mathbb{R}_{+}$, and $\lim _{t \rightarrow+\infty} \phi_{X}^{t}(x)=x_{0}$.

Proof. We choose for $U$ a convex globally hyperbolic neighbourhood of $x_{0}$, as in lemma 2. We suppose moreover that the time function $T: \rightarrow]-A, A[$ satisfies $x_{0} \in T^{-1}\{0\}$. Since $\forall x \in I_{U}^{+}\left[x_{0}\right], X(x) \neq 0$, we can suppose (looking at $-X$ instead of $X)$, that $\forall x \in I_{U}^{+}\left[x_{0}\right], X(x)$ is past-oriented. Now, let $x$ be in $I_{U}^{+}\left[x_{0}\right], T(x)=T_{0}$. The function $F: t \mapsto T\left(\phi_{X}^{t}(x)\right)$ is decreasing, and even strictly decreasing because since $X$ is causal, $d T\left(X\left(\phi_{X}^{t}(x)\right)\right)$ never vanishes (unless $X(x)=0$ ). What we claim is that the orbit $\phi_{X}^{t}(x)$ is trapped inside $I_{U}^{+}\left[x_{0}\right]$. Indeed, if for some $t_{0} \in \mathbf{R}, \phi^{t_{0}} \in U \backslash I_{U}^{+}\left[x_{0}\right]$, then there is $0 \leq t_{1} \leq t_{0}$ such that $\phi^{t_{1}} . x \in C_{U}^{+}\left[x_{0}\right]$. But this is impossible, since the $\phi_{X}^{t}$-orbits of points of $C_{U}^{+}\left[x_{0}\right]$ are (locally) contained in $C_{U}^{+}\left[x_{0}\right]$. So, the orbit $\phi_{X}^{t}(x)$ is in fact trapped in the compact subset $J_{U}^{+}\left[x_{0}\right] \cap\left\{x \in U, 0 \leq T(x) \leq T_{0}\right\}$. The function $F$ is decreasing and bounded from above, so that $\lim _{t \rightarrow+\infty} F(t)$ exists and $\lim _{t \rightarrow+\infty} F^{\prime}(t)=0$. Let $x_{\infty}$ be a limit point of some sequence $\left(\phi^{t_{k}} \cdot x\right)_{k \in \mathbb{N}}$. The remark above yields $d T\left(X\left(x_{\infty}\right)\right)=0$, so that $X\left(x_{\infty}\right)$ is tangent to an hypersurface $T=$ constant. Since these hypersurfaces are supposed to be spacelike and $X\left(x_{\infty}\right)$ is causal, we get $X\left(x_{\infty}\right)=0$, and finally $x_{\infty}=x_{0}$. The only accumulation point of $\left(\phi_{X}^{t}(x)\right)_{t \geq 0}$ is $x_{0}$, so that $\lim _{t \rightarrow+\infty} \phi_{X}^{t}(x)=x_{0}$, and the proof is completed.

Of course, we have the same statement if we replace $I_{U}^{+}\left[x_{0}\right]$ by $I_{U}^{-}\left[x_{0}\right]$.

\subsection{The case of a lightlike geodesic of singularities}

We now suppose that there is $U$, a convex globally hyperbolic neighbourhood of $x_{0}$, as in lemma 2 , such that $I=Z(X) \cap U$ is a lightlike geodesic segment. For all $x \in I_{U}^{+}\left[x_{0}\right]$, the set $J_{U}^{-}[x] \cap J_{U}^{+}\left[x_{0}\right]$ is compact. In particular, any lightlike segment of $C_{U}^{+}\left[x_{0}\right]$ intersects a lightlike segment of $C_{U}^{-}[x]$. Said in another way, $\forall x \in I_{U}^{+}\left[x_{0}\right]$, there is some $y \in I$ such that $x \in C_{U}^{+}[y]$. Moreover, since no lightlike geodesic triangles exist in $U$ (lemma 2), we get 
that this $y$ is unique, and denote it by $\pi(x)$. This defines a continuous projection $\pi: I_{U}^{+}\left[x_{0}\right] \rightarrow I$. The fiber $\pi^{-1}(\{y\})$ of $y \in I$ is just $C_{U}^{+}[y] \backslash I$.

Proposition 7. Changing $X$ into $-X$ if necessary, we have that $\forall x \in$ $I_{U}^{+}\left[x_{0}\right], \phi_{X}^{t}(x)$ is defined for all $t \in \mathbb{R}_{+}$, and $\lim _{t \rightarrow \infty} \phi_{X}^{t}(x)=\pi(x)$.

Proof. Since we supposed the dimension of $M$ is at least $3, U \backslash I$ is connected, and $X$ does not vanish on $U \backslash I$. So, changing $X$ into $-X$ if necessary, we will suppose that $X$ is past oriented on $U$. By what has been said above, given $x \in I_{U}^{+}\left[x_{0}\right]$, there is a lightlike geodesic segment $[\pi(x), x]$ joining $x$ and $\pi(x)$. This segment lies entirely in $U$, because $U$ is convex. Moreover, since $\phi_{X}^{t}$ fixes $\pi(x)$ and $D_{\pi(x)} \phi_{X}^{t}=I d \forall t \in \mathbf{R}$, we get that the orbit $\phi_{X}^{t}(x)$ is included in $[\pi(x), x]$. Remark that the only zeros of $X$ in $U$ are the points of $I$, so that $X$ does not vanish on $[\pi(x), x]$, and since it is past oriented, it "points" from $x$ to $\pi(x)$. It follows that $\lim _{t \rightarrow+\infty} \phi_{X}^{t}(x)=\pi(x)$, as claimed.

\section{Proof of Theorem 1}

\subsection{The case of an isolated singularity}

If we take $x \in I_{U}^{+}\left[x_{0}\right]$, Proposition 6 implies $\lim _{t \rightarrow+\infty}\left(I_{U}^{+}\left[x_{0}\right] \cap I_{U}^{-}\left[\phi_{X}^{t}(x)\right]\right)=$ $\lim _{t \rightarrow+\infty} \phi_{X}^{t}\left(I_{U}^{+}\left[x_{0}\right] \cap I_{U}^{-}[x]\right)=\left\{x_{0}\right\}$, what implies that all points of $I_{U}^{+}\left[x_{0}\right] \cap$ $I_{U}^{-}[x]$ are dynamically normal. Now proposition 3 yields:

Corollary 4. The sequence $\left(\phi^{k}\right)$ is stable at every point $x \in I_{U}^{+}\left[x_{0}\right]$.

If we are in dimension three, Proposition 5 directly implies Theorem 1. We now suppose that we are in dimension at least four.

Lemma 6. The Weyl tensor vanishes at $x_{0}$, and if $\operatorname{dim}(M)=3$, so does $C$.

Proof. We pick $y \in C_{U}^{+}\left[x_{0}\right]$, and choose a sequence $\left(y_{i}\right)$ of points of $I_{U}^{+}\left[x_{0}\right]$ converging to $y$. For all $i \in \mathbb{N}, \lim _{k \rightarrow+\infty} D_{y_{i}} \phi^{k}\left(X\left(y_{i}\right)\right)=0$, because of proposition 6 and the fact that $X\left(x_{0}\right)=0$. So, $X\left(y_{i}\right) \in H_{y_{i}}^{<<}$. If $X\left(y_{i}\right)$ is timelike, it means that $H_{y_{i}}^{<<}=T_{y_{i}} M$. In other words, $\left(\phi^{k}\right)_{k \in \mathbb{N}}$ is strongly stable at $y_{i}$, and $W_{y_{i}}=0$ by Proposition 4 . So, if $X\left(y_{i}\right)$ is timelike for infinitely many $i \in \mathbb{N}, W_{y}=0$. Now, since $\phi^{k}(y)$ tends to $x_{0}$ as $k$ goes to infinity, we get $W_{x_{0}}=0$.

It remains to study the case where $X\left(y_{i}\right)$ is lightlike for all, but a finite number of $i \in \mathbb{N}$. In this case, $H_{y_{i}}^{<<}$is nothing but the subspace $X\left(y_{i}\right)^{\perp}$, the orthogonal of $X\left(y_{i}\right)$ in $T_{y_{i}} M$ for $g_{y_{i}}$. It follows from proposition 4, point (ii), that $\operatorname{Im} W_{y_{i}} \subset X\left(y_{i}\right)^{\perp}$. We infer that $\operatorname{Im} W_{y} \subset X(y)^{\perp}$. We call $u(y)$ some nonzero vector of $T_{x_{0}} M$, which is tangent to the lightlike geodesic joining $x_{0}$ and $y$. Using the conformal invariance of the Weyl tensor under the action of $\phi^{k}$, we get $\operatorname{Im} W_{x_{0}} \subset u(y)^{\perp}$. But this is true for all $y$ in $C_{U}^{+}\left[x_{0}\right]$, and, since $\bigcap_{y \in C_{U}^{+}\left[x_{0}\right]} u(y)^{\perp}=\{0\}$, we get $W_{x_{0}}=0$, and the proof is complete. 
Now, Theorem 1 follows from the previous proposition and the point $(i)$ of Proposition 4.

\subsection{The case of a lightlike geodesic of singularities}

The continuity of $\pi$ and Proposition 7 imply that $\left(\phi^{k}\right)_{k \in \mathbb{N}}$ is dynamically normal at every point of $I_{U}^{+}\left[x_{0}\right]$ From proposition 3, we infer:

Corollary 5. The sequence $\left(\phi^{k}\right)_{k \in \mathbb{N}}$ is stable at every point $x \in I_{U}^{+}\left[x_{0}\right]$.

If we are in dimension three, Proposition 5 directly implies Theorem 1. We now suppose that we are in dimension at least four.

As in the case of an isolated singularity, we will be able to conclude the proof if we show that, for all $x \in I_{U}^{+}\left[x_{0}\right]$, the Weyl tensor vanishes at $\pi(x)$. In fact, it works essentially as above. Indeed, if some $y \in C_{U}^{+}[\pi(x)] \backslash I$ statisfies $W_{y}=0$, we are done, since $\lim _{t \rightarrow+\infty} \phi_{X}^{t}(y)=\pi(x)$ will imply $W_{\pi(x)}=0$. So, we suppose that the Weyl tensor is non zero at any point $y$ of $C_{U}^{+}[\pi(x)] \backslash I$. By Proposition 4, it means that $\operatorname{Im} W_{y}$ is a subspace of $X(y)^{\perp}$. Using $\lim _{t \rightarrow+\infty} \phi_{X}^{t}(y)=\pi(x)$, we get that $\operatorname{Im} W_{\pi(x)}$ is included in $u(y)^{\perp}$, where $u(y)$ is a vector of $T_{\pi(x)} M$, tangent to the lightlike geodesic joining $\pi(x)$ to $y$. Since this must be true for any $y \in C_{U}^{+}[\pi(x)] \backslash I$, we get once again $W_{\pi(x)}=0$.

\section{Some examples showing that Theorem 1 is sharp}

\subsection{About the compactification of Minkowski's space}

We recall here briefly how to compactify conformally Minkowski's space.

Let $\mathbf{R}^{2, n}$ be the space $\mathbf{R}^{n+2}$, endowed with the quadratic form $q^{2, n}(x)=$ $-2 x_{0} x_{n+1}-x_{2}^{2}+x_{3}^{2}+\ldots+x_{n}^{2}$. The isotropic cone of $q^{2, n}$ is the subset of $\mathbf{R}^{2, n}$ on which $q^{2, n}$ vanishes. We call $C^{2, n}$ this isotropic cone, with the origin removed. By $\pi$, we will mean the projection from $\mathbf{R}^{2, n}$ minus the origin, on $\mathbf{R} P^{n+1}$. The set $\pi\left(C^{2, n}\right)$ is a smooth hypersurface $\Sigma$ of $\mathbf{R} P^{n+1}$. This hypersurface turns out to be endowed with a natural Lorentzian conformal structure. Indeed, for any $x \in C^{2, n}$, the restriction of $q^{2, n}$ to the tangent space $T_{x} C^{2, n}$, that we call $\hat{q}_{x}^{2, n}$, is degenerate. Its kernel is just the kernel of the tangent map $d_{x} \pi$. Thus, pushing $\hat{q}_{x}^{2, n}$ by $d_{x} \pi$, we get a well defined Lorentzian metric on $T_{\pi(x)} \Sigma$. If $\pi(x)=\pi(y)$ the two Lorentzian metrics on $T_{\pi(x)} \Sigma$ obtained by pushing $\hat{q}_{x}^{2, n}$ and $\hat{q}_{y}^{2, n}$ are in the same conformal class. Thus, the form $q^{2, n}$ determines a well defined conformal class of Lorentzian metrics on $\Sigma$. One calls Einstein's universe the hypersurface $\Sigma$, together with this canonical conformal structure, and denote it by $\operatorname{Ein}_{n}$.

The projection on $\mathbf{E i n}_{n}$ of the intersection of $C^{2, n}$ with null 2-planes of $\mathbf{R}^{2, n}$ (resp. degenerate hyperplanes of $\mathbf{R}^{2, n}$ ) are called lightlike geodesics (resp. lightlike cones) of $\mathbf{E i n}_{n}$. The lightlike geodesics are closed curves, 
but one can not find a conformal parametrization, as defined in section 2.1, for these entire closed geodesics. One has to remove a point from them, to make it possible. The lightlike cones are the sets of lightlike geodesics passing through a common point $p$ (the vertex of the lightlike cone).

Let us identify Minkowski's space $\mathbf{R}^{1, n-1}$ with the subspace of $\mathbf{R}^{n+2}$ spanned by $e_{1}, . ., e_{n}$, and let us denote by $<,>$ the restriction of $q^{2, n}$ to $\operatorname{Vect}\left(e_{1}, \ldots, e_{n}\right)$. We define:

$$
\begin{aligned}
& \bar{s}: \mathbf{R}^{1, n-1} \rightarrow C^{2, n} \\
& x \mapsto<x, x>e_{0}+2 x+e_{n+1}
\end{aligned}
$$

Then the map $s=\pi \circ \bar{s}$ is a conformal embedding of $\mathbf{R}^{1, n-1}$ into $\mathbf{E i n}_{n}$. It is called stereographic projection of $\mathbf{R}^{1, n-1}$. The image $s\left(\mathbf{R}^{1, n-1}\right)$ is the complement in $\mathbf{E i n}_{n}$ of the lightlike cone with vertex $p_{\infty}=\pi\left(e_{0}\right)$. This cone is called cone at infinity and denoted by $C_{\infty}$. For a better understanding of the compactification of $\mathbf{R}^{1, n-1}$, the following lemma is useful (a proof is given in $[\mathrm{Fr} 1]$ p.53):

Lemma 7. After identifying $\mathbf{R}^{1, n-1}$ as an open subset of $\operatorname{Ein}_{n}$ thanks to the stereographic projection $s$, one has:

(i) Timelike and spacelike straightlines of $\mathbf{R}^{1, n-1}$ all converge to the point $p_{\infty}$ at infinity.

(ii) To each lightlike direction $u$ of $\mathbf{R}^{1, n-1}$ is associated a unique lightlike geodesic $\Delta_{u} \subset C_{\infty}$, and a map $\rho_{u}: \mathcal{D}_{u}$, to $\Delta_{u} \backslash\left\{p_{\infty}\right\}$ (where $\mathcal{D}_{u}$ is the space of straightlines with direction $u$ in $\mathbf{R}^{1, n-1}$ ), such that :

- any lightlike straightline $\Delta \in \mathcal{D}_{u}$ tends to $\rho_{u}(\Delta)$ at infinity.

- $\rho_{u}(\Delta)=\rho_{u}\left(\Delta^{\prime}\right)$ if and only if $\Delta$ and $\Delta^{\prime}$ are in the same degenerate affine hyperplane of $\mathbf{R}^{1, n-1}$.

Let us also say a few words about the group of conformal transformations of $\mathbf{E i n}_{n}$. From the very construction of $\mathbf{E i n}_{n}$, it is clear that the group $P O(2, n)$ acts naturally by conformal transformations on $\mathbf{E i n}_{n}$. It turns out that $P O(2, n)$ is the full conformal group of $\operatorname{Ein}_{n}$. Moreover, there is a Liouville theorem, asserting that any conformal transformation between connected open subsets of $\mathbf{E i n}_{n}$ is the restriction of a unique transformation of $P O(2, n)$. In particular, any conformal transformation of $\mathbf{R}^{1, n-1}$ extends in a unique way to a conformal transformation of $\mathbf{E i n}_{n}$. In particular, the conformal transformation $x \mapsto \frac{x}{\langle x, x\rangle}$, defined on $\mathbf{R}^{1, n-1} \backslash\{x \mid\langle x, x\rangle \neq 0\}$, can be seen as an element of $P O(2, n)$, that we call $\theta$. The transformation $\theta$ switch the points 0 and $p_{\infty}$, and the lightcone through 0 (in $\mathbf{E i n}_{n}$ ), with the lightcone through $p_{\infty}$.

\subsection{Two examples}

In what follows, we denote by $\mathbf{R}^{1, n-1}$ Minkowski's space, with $\langle x, x\rangle=$ $-x_{1}^{2}+x_{2}^{2}+\ldots+x_{n}^{2}$. We will call $g^{\text {can }}$ the metric $-d x_{1}^{2}+d x_{2}^{2}+\ldots+d x_{n}^{2}$. By $C^{1, n-1}$ (resp. $\Omega^{-}$, resp. $\Omega^{+}$), we will denote the set of lightlike (resp. 
timelike, resp. spacelike) vectors in $\mathbf{R}^{1, n-1}$.

1) Let $\phi_{X}^{t}$ be the translation $x \mapsto x+t e_{1}$ of $\mathbf{R}^{1, n-1}$, seen as a transformation of $P O(2, n)$. Using the properties stated in Lemma 7 , it is quite easy to check that $p_{\infty}$ is the unique fixed point of $\phi_{X}^{t}$, and that the orbits of $\phi_{X}^{t}$ are timelike in $\mathbf{R}^{1, n-1}$ and lightlike on $C_{\infty}$. Let $X$ the conformal vector field on $\mathbf{E i n}_{n}$, associated to $\phi_{X}^{t}$. The field $X$ is causal and has exactly one singularity, at $p_{\infty}$. We set $\psi_{Y}^{t}=\theta \circ \phi_{X}^{t} \circ \theta$ and $Y=\theta_{*} X$. It is clear that $Y$ is also a causal conformal vector field of $\mathbf{E i n}_{n}$, and that $0 \in \mathbf{R}^{1, n-1}$ is the unique singularity of $Y$. Any integral curve of $Y$ in $\mathbf{R}^{1, n-1}$ is parametrized by $t \mapsto a+t e_{1}$, $t \in \mathbb{R}$, for a unique $a \in C^{1, n-1}$ such that $\left\langle a, e_{1}\right\rangle \geq 0$. By an easy computation we get $\theta\left(a+t e_{1}\right)=\frac{a+t e_{1}}{t\left(2<a, e_{1}>-t\right)}$. Thus, the integral curves of $Y$ which are contained in $\Omega^{+}$are given by $t \mapsto \frac{a+t e_{1}}{t\left(2<a, e_{1}>-t\right)}$, for $\left.t \in\right] 0,2<a, e_{1}>[$. We call $H$ the hyperplane $\left\{x_{1}=0\right\}$. Since $<\theta\left(a+t e_{1}\right), e_{1}>=0$ exactly when $t=<a, e_{1}>$, we see that any integral curve of $Y$ in $\Omega^{+}$intersects $H$ at exactly one point. Moreover, since $H \backslash\{0\}$ is preserved by the conformal map $\theta, Y(x)$ is orthogonal to $H$ for any $x \in H \backslash\{0\}$.

On $\Omega^{+}$, we have two global coordinate systems. The first one is simply the restriction to $\Omega^{+}$of the "canonical" coordinates $\left(x_{1}, \ldots, x_{n}\right)$ on $\mathbf{R}^{1, n-1}$. The second one gives the coordinates $\left(y_{1}, \ldots, y_{n}\right)$ to the point $\psi_{Y}^{y_{1}} . y$, where $y=\left(0, y_{2}, \ldots, y_{n}\right) \in H \backslash\{0\}$. In these second coordinates, the action of the flow $\psi_{Y}^{t}$ is just given by $\left(y_{1}, y_{2}, \ldots, y_{n}\right) \mapsto\left(y_{1}+t, y_{2}, \ldots, y_{n}\right)$.

Let us define a metric $h^{\text {can }}$ on $\Omega^{+}$by the formula $h_{\psi_{Y}^{t} . y}^{\text {can }}\left(D_{y} \psi_{Y}^{t}(u), D_{y} \psi_{Y}^{t}(v)\right)=$ $\left.g_{y}^{c a n}(u, v), y \in H \backslash\{0\}, t \in\right] 0,2<a, e_{1}>$ [. This metric is clearly in the conformal class of $g^{\text {can }}$, and there is a smooth function $\lambda: \Omega^{+} \rightarrow \mathbb{R}$ such that $g^{\text {can }}=e^{\lambda} h^{\text {can }}$ in $\Omega^{+}$. In the coordinates $\left(y_{1}, \ldots, y_{n}\right)$, this metric writes as $h^{\text {can }}(y)=-e^{\nu^{\text {can }}} d y_{1}^{2}+d y_{2}^{2}+\ldots+d y_{n}^{2}$, where $\nu^{\text {can }}$ is a smooth function on $\Omega^{+}$depending only of $y_{2}, . ., y_{n}$. For any family $\sigma=\left(\nu_{i}\right)_{2 \leq i \leq n}$ of smooth functions on $\Omega^{+}$, depending only of $y_{2}, \ldots, y_{n}$, we associate the Lorentzian metric $h^{\sigma}(y)=-e^{\nu^{c a n}} d y_{1}^{2}+e^{\nu_{2}(y)} d y_{2}^{2}+\ldots+e^{\nu_{n}(y)} d y_{n}^{2}$ on $\Omega^{+}$. For any choice of such family $\sigma, Y$ is a Killing vector field for $h^{\sigma}$.

For every $r \in \mathbb{R}$, and $x \in \mathbf{R}^{n}$, we call $B(x, r)$ the open Euclidean ball of radius $r$ centered at $x$, and $\dot{B}(x, r)$ this same ball with $x$ removed. For every $k \in \mathbb{N}^{*}$,we call $\Lambda(k)$ the intersection of $B(0, k)$ with the union of all orbits $\psi_{Y}^{t} . x, x \in \dot{B}\left(0, \frac{1}{k}\right) \cap H$. Let $\Sigma$ be the set of families $\sigma=\left(\nu_{i}\right)_{2 \leq i \leq n}$, such that for all $k \in \mathbb{N}^{*}$, and $i, j, l_{1}, \ldots, l_{m} \in\{1, \ldots, n\}$ with $1 \leq m \leq k$ :

$$
\sup _{x \in \Lambda(k)}\left|e^{\lambda(x)} h_{i j}^{\sigma}(x)-e^{\lambda} h_{i j}^{c a n}\right|<\frac{1}{k}
$$

and 


$$
\sup _{x \in \Lambda(k)}\left|\frac{\partial^{m}\left(e^{\lambda} h_{i j}^{\sigma}\right)}{\partial x_{l_{1}} \ldots \partial x_{l_{m}}}(x)-\frac{\partial^{m}\left(e^{\lambda} h_{i j}^{c a n}\right)}{\partial x_{l_{1}} \ldots \partial x_{l_{m}}}(x)\right|<\frac{1}{k}
$$

Notice that we are dealing here with the coordinates of the metric components in the system $\left(x_{1}, \ldots, x_{n}\right)$. The set $\Sigma$ is clearly non empty (it contains the family $\left.\nu_{2}=\ldots=\nu_{n}=0\right)$.

Let $M$ denote the space $\mathbf{R}^{n}$. For every $\sigma \in \Sigma$, we define a Lorentzian metric $\bar{h}^{\sigma}$ on $M$ by $\bar{h}^{\sigma}(x)=e^{\lambda} h^{\sigma}(x)$ for $x \in \Omega^{+}$, and $\bar{h}^{\sigma}(x)=g^{\text {can }}$ elsewhere. Then, the metric $\bar{h}^{\sigma}$ is smooth on $M$ and $Y$ is a causal conformal vector field of $\left(M, \bar{h}^{\sigma}\right)$. The smoothness of $\bar{h}^{\sigma}$ on $\Omega^{+} \cup \Omega^{-}$is obvious, but it remains to show that $\bar{h}^{\sigma}$ is smooth at every point of $C^{1, n-1}$. Let $x_{0} \in C^{1, n-1}$ be such a point. There is a map $k \mapsto n_{k}$, defined for $k \geq K$ big enough, with $n_{k} \in \mathbb{N}$, such that $\left(B\left(x_{0}, \frac{1}{k}\right) \cap \Omega^{+}\right) \subset \Lambda\left(n_{k}\right)$ but $\left(B\left(x_{0}, \frac{1}{k}\right) \cap \Omega^{+}\right) \not \subset$ $\Lambda\left(n_{k}+1\right)$. Since the orbits of $\psi_{Y}^{t}$ close to $C^{1, n-1}$ hit $H$ close to $\{0\}$, we have $\lim _{k \rightarrow+\infty} n_{k}=+\infty$. Now, observe that $\lim _{k \rightarrow+\infty}\left(\sup _{x \in B\left(x_{0}, \frac{1}{k}\right)} \mid \bar{h}_{i j}^{\sigma}(x)-\right.$ $g_{i j}^{c a n}(x) \mid=\lim _{k \rightarrow+\infty}\left(\sup _{x \in \Lambda\left(n_{k}\right)}\left|e^{\lambda(x)} h_{i j}^{\sigma}(x)-g_{i j}^{c a n}(x)\right|\right)=0$, because of condition (1) determinig $\Sigma$. Thus, the metric $\bar{h}^{\sigma}$ is continuous at $x_{0}$.

We fix an open interval $]-\epsilon,+\epsilon[$, with $\epsilon$ small. For $j \in\{1, \ldots, n\}$, the only $t \in]-\epsilon, \epsilon\left[\right.$ such that $t \mapsto x_{0}+t e_{s}$ cuts $C^{1, n-1}$ is exactly $t=0$. To prove the smoothness of the metric, we must prove that for any $m \in \mathbb{N}^{*}$, and any $\left.l_{1}, \ldots, l_{m}, s \in\{1, \ldots, n\}, \lim _{t \rightarrow 0} \frac{\partial^{m}\left(\bar{h}_{j}^{\sigma}\right)}{\partial x_{l_{1}} \ldots \partial x_{l_{m}}}\left(x_{0}+t e_{s}\right)\right)$ exists.

By the previous remark, $x_{0}+t \epsilon \in \Omega^{-}$for every $\left.t \in\right]-\epsilon, 0\left[\right.$, or $x_{0}+t \epsilon \epsilon$ $\Omega^{+}$for every $\left.t \in\right]-\epsilon, 0\left[\right.$. In the first case, $\left.\frac{\partial^{m}\left(\bar{h}^{\sigma}\right)}{\partial x_{l_{1}} \ldots \partial x_{l_{m}}}\left(x_{0}+t e_{s}\right)\right)=0$ for every $t \in]-\epsilon, 0\left[\right.$ and we get $\left.\lim _{t \rightarrow 0^{-}} \frac{\partial^{m}\left(\bar{h}^{\sigma}\right)}{\partial x_{l_{1}} \ldots \partial x_{l_{m}}}\left(x_{0}+t e_{s}\right)\right)=0$. If we are in the second case, then for every $k \in \mathbb{N}^{*}$, there is a $\left.t_{k} \in\right]-\epsilon, 0[$ such that $x_{0}+t e_{s} \in \Lambda(k)$ as soon as $\left.t \in\right] t_{k}, 0[$. Since $\sigma$ was chosen in $\Sigma$, we get by condition 2 that $\left.\sup _{t \in] t_{k}, 0[} \mid \frac{\partial^{m}\left(\bar{h}^{\sigma}\right)}{\partial x_{l_{1}} \ldots \partial x_{l_{m}}}\left(x_{0}+t e_{s}\right)\right) \mid<\frac{1}{k}$. We get once again: $\left.\lim _{t \rightarrow 0^{-}} \mid \frac{\partial^{m}\left(\bar{h}^{\sigma}\right)}{\partial x_{l_{1}} \ldots \partial x_{l_{m}}}\left(x_{0}+t e_{s}\right)\right) \mid=0$.

We prove in the same way that $\left.\lim _{t \rightarrow 0^{+}} \mid \frac{\partial^{m}\left(\bar{h}^{\sigma}\right)}{\partial x_{l_{1}} \ldots \partial x_{l_{m}}}\left(x_{0}+t e_{s}\right)\right) \mid=0$, and we conclude that for every $m \in \mathbb{N}^{*}$, and any $l_{1}, \ldots, l_{m}, j \in\{1, \ldots, n\}$, $\left.\lim _{t \rightarrow 0} \frac{\partial^{m}\left(\bar{h}^{\sigma}\right)}{\partial x_{l_{1}} \ldots \partial x_{l_{m}}}\left(x_{0}+t e_{s}\right)\right)=0$.

Now, the metric $-e^{\nu^{c a n}} d y_{1}^{2}+e^{\nu_{2}} d y_{2}^{2}+\ldots+e^{\nu_{n}} d y_{n}^{2}$ is in the conformal class of $-d y_{1}^{2}+e^{\nu_{2}-\nu^{c a n}} d y_{2}^{2}+\ldots+e^{\nu_{n}-\nu^{c a n}} d y_{n}^{2}$, which is conformally flat only when $e^{\nu_{2}-\nu^{c a n}} d y_{2}^{2}+\ldots+e^{\nu_{n}-\nu^{c a n}} d y_{n}^{2}$ has constant curvature. In fact, for a generic choice of $\sigma=\left(\nu_{i}\right)_{2 \leq i \leq n} \in \Sigma$, the Weyl tensor (or the Cotton tensor if $n=3$ ) is nowhere vanishing. For such a choice $\sigma_{0} \in \Sigma$, the previous construction yields a smooth Lorentz manifold $\left(M, \bar{h}^{\sigma_{0}}\right)$, with a causal vector field $Y$, admitting a unique singularity $x_{0}=0$, and such that the Weyl tensor of $\bar{h}^{\sigma_{0}}$ (resp. the Cotton tensor if $n=3$ ), vanishes exactly on the closure of 
$I^{+}\left[x_{0}\right] \cup I^{-}\left[x_{0}\right]$. The conclusion $(i)$ of Theorem 1 is thus optimal.

2) For our second example, we consider $\phi_{X}^{t}$, the translation of vector $t \tau=$ $t\left(e_{1}+e_{2}\right)$ (we see this translation as an element of $P O(2, n)$ ). Using again the properties stated in Lemma 7 , one checks that the orbits of $\phi_{X}^{t}$ are all lightlike, and that the fixed points of $\phi_{X}^{t}$ are exactly the points of the lightlike geodesic $\Delta_{\tau} \subset C_{\infty}$. The conformal vector field $X$ associated to $\phi_{X}^{t}$ is causal, and its singularities are the points of $\Delta_{\tau}$. As previously, we introduce $\psi_{Y}^{t}=\theta \circ \phi_{X}^{t} \circ \theta$, and $Y=\theta_{*} X$. The singularities of $Y$ in $\mathbf{R}^{1, n-1}$ are exactly the points of the straightline $\mathbb{R} . \tau$. The integral curves of $Y$ in $\mathbf{R}^{1, n-1}$ are the (open) lightlike halflines with endpoint on $\mathbb{R} . \tau$. We call $p=e_{1}, q=-e_{1}$, and take for manifold $M$ the intersection $I^{+}[q] \cap I^{-}[p]$ (the future and past sets are taken in $\mathbf{R}^{1, n-1}$ ). This is a "diamond like" open subset of $\mathbf{R}^{1, n-1}$. The line $\mathbb{R} . \tau$ intersects $M$ along an open interval $I_{\tau}$, with endpoints $a$ and $b$. We choose $a$ to be in the future of $b$. Let $V$ be the intersection $\left(I^{-}[a] \cap I^{+}[q]\right) \cup\left(I^{+}[b] \cap I^{-}[p]\right)$. This is an open subset of $M$, characterized by the property that every integral curve of $Y$ in $V$, has an endpoint on $I_{\tau}$. We define $\Omega$ to be the open subset $M \backslash \bar{V}$. If, as previously, $H$ denotes the hyperplane $x_{1}=0$, we call $B=H \cap \Omega$. Then any integral curve of $Y$ in $\Omega$ intersect $B$ at exactly one point. This allows us to consider two coordinate systems on $\Omega$. The first one is just the restriction to $\Omega$ of the canonical coordinates $\left(x_{1}, \ldots, x_{n}\right)$ on $\mathbf{R}^{1, n-1}$. The second one, defined on $\Omega^{\prime} \subset \mathbf{R}^{n}$, gives coordinates $\left(y_{1}, \ldots, y_{n}\right)$ to the point $\psi_{Y}^{y_{1}} \cdot \theta . y$, for $y=\left(0, y_{2}, \ldots, y_{n}\right) \in \theta$.B.

Let us choose a sequence $\left(B_{k}\right)_{k \in \mathbb{N}}$ of open subsets of $B$, having compact closure in $B$, such that $\bar{B}_{k} \subset B_{k+1}$ for all $k$, and $B=\bigcup_{k \in \mathbb{N}} B_{k}$. Then, if $U_{k}$ denotes the saturation of $B_{k}$ by the orbits of $\psi_{Y}^{t}$ in $\Omega$, then $\Omega=\bigcup_{k \in \mathbb{N}} U_{k}$. In the following, we will write $U_{k}^{c}$ for the complementary of $U_{k}$ in $\Omega$.

For every smooth function $\sigma: \Omega^{\prime} \rightarrow \mathbb{R}$, depending only of $y_{3}$, we associate the Lorentzian metric $h^{\sigma}$ on $\Omega$, defined in terms of coordinates $\left(y_{1}, \ldots, y_{n}\right)$ by $h^{\sigma}(y)=e^{\sigma\left(y_{3}\right)} d y_{1} d y_{2}+\sum_{k=2}^{n} d y_{k}^{2}$. For any $\sigma, Y$ is a Killing field for $h^{\sigma}$ on $\Omega$. When $\sigma$ is identically zero, we get a metric $h^{\text {can }}$ on $\Omega$, which is in the same conformal class as $g^{\text {can }}$ (because $\theta$ is conformal). Thus there exists a smooth function $\lambda: \Omega \rightarrow \mathbb{R}$, such that $e^{\lambda} h^{c a n}=g^{\text {can }}$. We can check that $e^{\lambda(x)}=\frac{1}{\langle x, x\rangle}$. As in the previous example, we introduce the set $\Sigma$ of smooth $\sigma=\sigma\left(y_{3}\right)$, satisfying, for all $k \in \mathbb{N}^{*}, i, j, l_{1}, \ldots, l_{m} \in\{1, \ldots, n\}$ and $1 \leq m \leq k$

$$
\sup _{x \in U_{k}^{c}}\left|e^{\lambda} h_{i j}^{\sigma}(x)-e^{\lambda} h_{i j}^{c a n}(x)\right|<\frac{1}{k}
$$




$$
\sup _{x \in U_{k}^{c}}\left|\frac{\partial^{m}\left(e^{\lambda} h_{i j}^{\sigma}\right)}{\partial x_{l_{1}} \ldots x_{l_{m}}}(x)-\frac{\partial^{m}\left(e^{\lambda} h_{i j}^{c a n}\right)}{\partial x_{l_{1}} \ldots \partial x_{l_{m}}}(x)\right|<\frac{1}{k}
$$

Given $\sigma \in \Sigma$, we define a Lorentzian metric $\bar{h}^{\sigma}$ on $M$ by $\bar{h}^{\sigma}(x)=e^{\lambda} h^{\sigma}(x)$ for $x \in \Omega$, and $\bar{h}^{\sigma}(x)=g^{\text {can }}$ elsewhere. By a proof similar to that of the first example, we get that the metric $\bar{h}^{\sigma}$ is smooth on $M$ and $Y$ is a causal conformal vector field of $\left(M, \bar{h}^{\sigma}\right)$.

For a metric $e^{\sigma\left(y_{3}\right)} d y_{1} d y_{2}+\sum_{k=2}^{n} d y_{k}^{2}$, we can do a few computations. We get that all Christofel symbols are zero, except $\Gamma_{32}^{1}=\Gamma_{23}^{1}=-\frac{1}{2} \sigma^{\prime} e^{-\sigma}$, $\Gamma_{13}^{1}=\Gamma_{31}^{1}=\frac{1}{2} \sigma^{\prime}, \Gamma_{23}^{2}=\Gamma_{32}^{2}=\frac{1}{2} \sigma^{\prime}$ and $\Gamma_{12}^{3}=\Gamma_{21}^{3}=-\frac{1}{2} \sigma^{\prime} e^{\sigma}$. Using the formula $R_{i l k}^{l}=\partial_{i} \Gamma_{l k}^{l}-\partial_{l} \Gamma_{i k}^{l}+\Sigma_{r=1}^{n}\left(\Gamma_{l k}^{r} \Gamma_{i r}^{l}-\Gamma_{i k}^{r} \Gamma_{l r}^{l}\right)$, we find that :

$R_{212}^{1}=R_{232}^{3}=\frac{1}{4}\left(\sigma^{\prime}\right)^{2}$.

$R_{132}^{3}=\frac{e^{\sigma}}{2}\left(\sigma^{\prime \prime}+\frac{1}{2}\left(\sigma^{\prime}\right)^{2}\right)$.

$R_{313}^{1}=R_{323}^{2}=\frac{1}{2} \sigma^{\prime \prime}+\frac{1}{4}\left(\sigma^{\prime}\right)^{2}$.

This gives three nonzero components for the Ricci tensor, namely:

$R_{12}=\frac{e^{\sigma}}{2}\left(\sigma^{\prime \prime}+\frac{1}{2}\left(\sigma^{\prime}\right)^{2}\right)$.

$R_{22}=\frac{1}{2}\left(\sigma^{\prime}\right)^{2}$.

$R_{33}=\sigma^{\prime \prime}+\frac{1}{2}\left(\sigma^{\prime}\right)^{2}$.

We infer that the scalar curvature of this metric is $S=\left(\frac{e^{\sigma}}{2}+1\right)\left(\sigma^{\prime \prime}+\right.$ $\left.\frac{1}{2}\left(\sigma^{\prime}\right)^{2}\right)$.

In local coordinates, the components of the Cotton tensor are given by: $C_{i j k}=\nabla_{k} R_{i j}-\nabla_{j} R_{i k}+\frac{1}{2(n-1)}\left(g_{i k} \partial_{j} S-g_{i j} \partial_{k} S\right)$.

In particular, we get $C_{223}=\sigma^{\prime} \sigma^{\prime \prime}+\frac{1}{2(n-2)} \frac{d}{d y_{3}}\left(\left(\frac{e^{\sigma}}{2}+1\right)\left(\sigma^{\prime \prime}+\frac{1}{2}\left(\sigma^{\prime}\right)^{2}\right)\right)$.

Now, let us remark that there exists a sequence $\epsilon_{k}$, such that $\sigma \in \Sigma$ as soon as $\max _{1 \leq m \leq k}\left(\sup _{y \in B(0, k)^{c} \cap \Omega^{\prime}}\left|\sigma^{(m)}\left(y_{3}\right)\right|\right)<\epsilon_{k}$ for every $k \in \mathbb{N}(B(0, k)$ stands here for the Euclidean ball of radius $k$ centered at 0$)$. So, we can choose smooth functions $\sigma$ such that $\sigma \in \Sigma$ and $C_{223}(y)$ never vanishes on $\Omega^{\prime}$. For such choices of $\sigma$, we get smooth Lorentz manifolds $\left(M, \bar{h}^{\sigma}\right)$, with $Y$ as conformal vector field, such that the Weyl tensor (resp. the Cotton tensor in dimension 3) vanishes exactly on the closure of $V=\bigcup_{x \in I_{\tau}} I^{+}[x] \cup I^{-}[x]$. The conclusion (ii) of Theorem 1 is thus optimal.

\section{Remark.}

In this article, we did not make any compactness asumtion on the manifold $(M, g)$. In the compact case, it is likely that a stronger conclusion than that of Theorem 1 must hold. Namely, the presence of a nontrivial causal conformal vector field, vanishing somewhere on a compact Lorentz manifold should ensure that the manifold is conformally flat. Nevertheless, the global dynamical behaviour of causal conformal vector fields on compact manifolds seems not so easy to understand. For instance, in [Fr2], section 4.5 and 4.6, quite complicated examples are constructed (these examples are conformally flat). 
Aknowledgments: I would like to thank the Institut für Mathematik of the Humboldt-Universität in Berlin, and especially Professor Helga Baum, for kindly explaining to me basic facts about twistor-spinors, and their link to conformal geometry.

\section{References}

[A] D. AleKseEvsKi - Self-similar Lorentzian manifolds. Ann. Global Anal. Geom. 3 (1985), no. 1, 59-84.

[BL] H. BAum, F. Leitner - The twistor equation in Lorentzian Spin geometry. Math. Z. 247 (2004), no. 4, 795-812.

[BE] J.K. BeEm, P.E. Ehrlich - Global Lorentzian Geometry. Dekker, New York, 1981.

[B] A.Besse - Einstein manifolds. Ergebnisse der Mathematik und ihrer Grenzgebiete (3), 10. Springer-Verlag, Berlin, 1987.

[E] L.Eisenhart - Riemannian Geometry. 2d printing. Princeton University Press, Princeton, N. J., 1949.

[Fr1] C.Frances - Géométrie et dynamique lorentziennes conformes. Thèse. Available at http : //mahery.math.u - psud.fr/ frances.

[Fr2] C.Frances - Sur les variétés lorentziennes dont le groupe conforme est essentiel. Math. Ann. 332 (2005), no 1, 103-119.

[KR1] W. KÜHNEL, H.B RADEMACHER - Essential conformal fields in pseudo-Riemannian geometry I. J. Math. Pures Appl. (9) 74 (1995), no. 5, 453-481.

[KR2] W. KüHNEL, H.B RADEMACHeR - Essential conformal fields in pseudo-Riemannian geometry II. J. Math. Sci. Univ. Tokyo 4 (1997), no. 3, 649-662.

[LM] H.B Lawson, M.Michelsohn - Spin geometry. Princeton Mathematical Series, 38. Princeton University Press, Princeton, NJ, 1989.

[L] F. LEITNER - Zeros of conformal vector fields and twistor spinors in Lorentzian geometry. SFB288-Preprint. No 439. Berlin 1999

[M] M.J. Markowitz - An intrinsic conformal Lorentz pseudodistance. Math. Proc. Camb. Phil. Soc, no 89 (1981), 359-371. 
[Z] A. ZEGHIB - Isometry groups and geodesic foliations of Lorentz manifolds. I,II. Foundations of Lorentz dynamics. Geom. Funct. Anal. 9 (1999), no. 4, 775-822.

Charles FRANCES

Département de Mathématiques

Université Paris-Sud

91405 ORSAY Cedex

Charles.Frances@math.u-psud.fr 